\title{
Star Formation in Cold, Spherical, Magnetized Molecular Clouds
}

\author{
Pedro N. Safier ${ }^{1}$, Christopher F. McKee ${ }^{2}$, and Steven W. Stahler ${ }^{3}$ \\ University of California at Berkeley \\ Department of Astronomy \\ Berkeley CA 94720-3411
}

\begin{abstract}
We present an idealized, spherical model of the evolution of a magnetized molecular cloud due to ambipolar diffusion. This model allows us to follow the quasi-static evolution of the cloud's core prior to collapse and the subsequent evolution of the remaining envelope. By neglecting the thermal pressure gradients in comparison with magnetic stresses and by assuming that the ion velocity is small compared with the neutral velocity, we are able to find exact analytic solutions to the MHD equations. We show that, in the case of a centrally condensed cloud, a core of finite mass collapses into the origin leaving behind a quasi-static envelope, whereas initially homogeneous clouds never develop any structure in the absence of thermal stresses, and collapse as a whole. Prior to the collapse of the core, the cloud's evolution is characterized by two phases: a long, quasi-static phase where the relevant timescale is the ambipolar diffusion time (treated in this paper), and a short, dynamical phase where the characteristic timescale is the free-fall time. The collapse of the core is an "outside-in" collapse. The quasi-static evolution terminates when the cloud becomes magnetically supercritical; thereafter its evolution is dynamical, and a singularity develops at the origin - a protostar. After the initial formation of the protostar, the outer envelope continues to evolve quasi-statically, while the region of dynamical infall grows with time - an "inside-out" collapse. We use our solution to estimate the magnetic flux trapped in the collapsing core and the mass accretion rate onto the newly formed protostar.

Our results agree, within factors of order unity, with the numerical results of Fiedler \& Mouschovias (1992) for the physical quantities in the midplane of a collapsing, magnetized, axisymmetric cloud up to the onset of dynamical collapse. Our simple approach thus captures the basic physics of a self-gravitating, magnetized cloud in which the evolution is driven by ambipolar diffusion. It also enables us to treat the evolution of the accretion onto the protostar after collapse, for which detailed numerical results are as yet unavailable. Remarkably, we find that at late times the
\end{abstract}

\footnotetext{
${ }^{1}$ Current address: University of Maryland at College Park, Department of Astronomy, College Park MD 20742; e-mail: safier@astro.umd.edu

${ }^{2}$ Physics Department, University of California at Berkeley; cmckee@astro.berkeley.edu

3stahler@astron.berkeley.edu
} 
accretion rate becomes comparable to that of a non-magnetized, singular isothermal sphere, provided that the ionization is due to galactic cosmic rays.

Subject headings: diffusion-hydromagnetics - ISM: clouds - ISM: magnetic fieldsplasmas - stars: formation

\section{Introduction}

How do stars form? This age-old question is one of the outstanding challenges of modern astrophysics. Even though we still lack a definite answer to this question, some partial answers have begun to emerge over the last three decades. The birth of molecular radio astronomy and infrared astronomy enabled us to definitively identify molecular clouds as the site of star formation, and to probe the physical conditions therein (Zuckerman \& Palmer, 1974). Notwithstanding the spectacular advances in instrumentation of the last decade and the resulting explosive growth in the number of infrared and radio observations, the detection of a cloud undergoing dynamical collapse is very difficult; to date there are only a few, tentative such detections (see Zhou et al., 1994 and references therein). By necessity, then, one has to resort to theory to understand how the molecular gas ultimately condenses into a star.

The efforts in this direction can be traced back to the pioneering work of Larson (1969) and Penston (1969). In these early calculations only thermal pressure - and sometimes rotation - were included, while magnetic fields were neglected (see Mouschovias [1978] and Bodenheimer \& Black [1978] for a list of early references). Of particular importance are the semi-analytic similarity solutions of Shu (1977) and Hunter (1977). These spherical solutions, though idealized, shed important light on the nature of the non-magnetic collapse. Non-magnetic collapse calculations are still pursued today; the current state of development in this field is well represented by the work of Myhill \& Boss (1993), Boss \& Myhill (1993), and Foster \& Chevalier (1993).

The relevance of magnetic fields to star formation were first pointed out by Mestel \& Spitzer (1956). Using the virial theorem, Strittmater (1966) calculated the critical mass for gravitational collapse perpendicular to the field lines of a cold, uniformly magnetized cloud, and Field (1965) studied the effect of the magnetic field on the thermal instability. These studies established that the presence of a magnetic field introduces qualitative changes to the conclusions of non-magnetic studies. However, not until the late seventies were detailed numerical calculations of magnetic clouds carried out. Mouschovias (1976a,b) performed the first self-consistent numerical studies of equilibrium, self-gravitating magnetic clouds, and Scott \& Black (1978) were the first to study the dynamics of collapsing, non-rotating clouds with a frozen-in magnetic field. With the introduction of a magnetic field, a realistic description requires at least two spatial dimensions. Moreover, at the densities of interest $\left(n \gtrsim 10^{3} \mathrm{~cm}^{-3}\right)$ the degree of ionization is low, and one has to consider the ion and neutral material as two separate fluids. This two-fluid nature of the problem has 
important consequences for the stability and collapse of magnetized clouds. The drift of the neutrals relative to the ions - a process known as ambipolar diffusion (Mestel \& Spitzer, 1956) - is responsible for bringing to the verge of collapse an otherwise stable cloud. As ambipolar diffusion proceeds, the density of the central region grows until the inner core attains a mass larger than a critical mass. Once this happens, the central region of the cloud is in a state of dynamical collapse (for a review, see Mouschovias [1996] and McKee et. al [1993] and references therein).

Except for some idealized, one-dimensional solutions which illustrated the role played by ambipolar diffusion in the redistribution of magnetic flux (Mouschovias 1979; Shu 1983; Scott, 1984), the study of the stability and collapse of realistic, magnetized clouds has been the domain of complex numerical calculations, with major contributions by Nakano (1979, 1982, 1983); Mouschovias and collaborators (Mouschovias, Paleologou, \& Fiedler 1985; Mouschovias \& Morton, 1991; Fiedler \& Mouschovias, 1992; Basu \& Mouschovias, 1994, 1995a,b; Ciolek \& Mouschovias, 1993, 1994a,b, 1995); Tomisaka, Ikeuchi, \& Nakamura (1988a,b; 1989; 1990); Tomisaka, (1991); Lizano \& Shu (1989); and Galli \& Shu (1993a,b). In contrast to the non-magnetic models, none of these calculations can follow the cloud's evolution up to and beyond the point where a star is formed. They either stop at an early stage of dynamical collapse (Fiedler \& Mouschovias, 1992); or tackle the problem after the formation of the star and follow the infall of the envelope under the gravitational field of a point source (Galli \& Shu, 1993b).

The shortcomings of current numerical work, and the lack of analytic or semi-analytic models to complement and illuminate the numerical results, motivated us to undertake the present study. Our goal is to understand - by means of a very simple model - how a globally stable cloud evolves, through ambipolar diffusion, into a state where a central core collapses, and to shed some light on the evolution of the remnant envelope. By doing so, we hope to distill the basic features that can be lost in the detail and complexity of numerical calculations. Previous attempts along these lines adopted either planar (Shu, 1983; Paleologou \& Mouschovias, 1983) or cylindrical symmetry (Mouschovias \& Morton, 1991). These clouds are always magnetically subcritical under conditions of flux freezing, and are of limited relevance to the study of collapsing clouds (Mouschovias, 1991; McKee et al., 1993). In our case, we adopt spherical symmetry, where the only non-vanishing component of the Lorentz force is the scalar term $\propto d B^{2} / d r$. In contrast to the planar and cylindrical cases, we cannot identify the actual magnetic field lines, but a three-dimensional cloud can undergo collapse even when flux freezing holds (Mouschovias, 1991; McKee et al., 1993).

In this paper, we neglect thermal pressure altogether, and set the gas temperature equal to zero. The case where thermal support is included is deferred to a second paper. Our motivation for this drastic approximation is twofold. From a practical viewpoint, this simplification enables us to treat ambipolar diffusion from the onset of cloud contraction to the collapse of a stellar core and beyond, an intrinsically non self-similar problem. Second, we show explicitly ( 55.2 .2$)$ that the role of thermal pressure diminishes in time as the central density rises. Thus, our calculation should give an increasingly accurate representation of the cloud's later evolution, as it forms a protostar and then slowly drains onto the protostar. 
The paper is organized as follows: in $\S 2$ we introduce our basic equations and approximations and present a general solution; the general properties of the solution for a particular family of initial conditions are presented in $\S 3$; in $\S 4$ we study the collapse of a homogeneous cloud; $\S 5$ contains our results for a centrally condensed cloud and a comparison between our results and the numerical results of Fiedler \& Mouschovias (1992); and our conclusions are presented in $\S 6$.

\section{Formulation and a General Solution}

We start with the general, two-fluid MHD equations governing ambipolar diffusion in self-gravitating molecular clouds (see, e.g., Paleologou \& Mouschovias, 1983). We assume steady-state ionization - a situation that obtains in dense clouds - and adopt the ionization law

$$
n_{i}=K_{i} n_{n}^{1 / 2}
$$

where $n_{i}$ and $n_{n}$ are, respectively, the ion and neutral number density sand $K_{i}$ is a constant with value $K_{i}=9.5 \times 10^{-6} \mathrm{~cm}^{-3 / 2}$ when $\mathrm{HCO}^{+}$is the most abundant ion (Elmegreen, 1979).

Our first basic approximation is that thermal stresses are negligible compared to magnetic forces, and we set the temperature of the gas equal to zero. The physical regime where this approximation is likely to be appropriate can be evaluated with the help of the parameter

$$
\beta=\frac{8 \pi k_{\mathrm{B}} n_{n} T}{B^{2}}
$$

where $k_{\mathrm{B}}$ is Boltzmann's constant, $T$ is the gas temperature, and $B$ is the strength of the magnetic field. For typical conditions in a starless cloud (see, e.g., Cernicharo, 1991; Heiles, 1987; Myers, 1985)

$$
\beta=0.04\left(\frac{n_{n}}{10^{3} \mathrm{~cm}^{-3}}\right)\left(\frac{T}{10 \mathrm{~K}}\right)\left(\frac{B}{30 \mu \mathrm{G}}\right)^{-2} .
$$

Therefore, low-mass clumps are magnetically dominated prior to the point at which their density is high enough for them to be visible as ammonia cores. The line widths of more massive clumps are often supersonic, so thermal pressure is relatively small there as well. However, we have not explicitly included turbulent pressure in our models because, in this formulation, its effects could be incorporated only under the artificial assumption that it is proportional to the static magnetic pressure.

To further simplify the problem we impose spherical symmetry. The accuracy of this approximation is unclear. Theoretical models of cloud cores suggest that the cores are flattened along field lines (see, eg., Fiedler \& Mouschovias, 1992; Lizano \& Shu, 1989; Tomisaka, Ikeuchi, \& Nakamura, 1988a). The observational evidence on cloud shapes has been interpreted as indicating that they are prolate (Myers et. al, 1991; Ryden, 1996) or that they are indeed flattened along field lines but that they are toroidal (Li \& Shu 1996). Physically, the thermal and turbulent pressure, which we have neglected, prevent actual cloud cores from becoming too flattened, and 
therefore we expect that our model will be qualitatively correct. Spherically symmetric models of magnetized clouds have been shown to represent the conditions for the onset of gravitational collapse reasonably well (Mouschovias \& Spitzer, 1976; Holliman \& McKee, 1996). Because we impose spherical symmetry, we cannot identify the actual magnetic field lines, nor can we account for nonradial forces. However, since we shall follow only the quasi-static part of the collapse (see below), we need the force equation only to estimate the flux remaining in the cloud, not to determine the dynamics of the collapse. As a result, we can adopt a simple approximation for the magnetic force, keeping the pressure gradient associated with the tangential fields but ignoring the associated tension. Let $\mathbf{B}_{t} \equiv \boldsymbol{B}_{\theta}+\boldsymbol{B}_{\phi}$ be the tangential field. The radial component of the magnetic force is then

$$
F_{r}=\frac{1}{4 \pi}\left(-\frac{1}{2} \frac{\partial B_{t}^{2}}{\partial r}+\mathbf{B}_{t} \cdot \nabla B_{r}-\frac{B_{t}^{2}}{r}\right) .
$$

The last term represents the hoop stress, an inward force due to magnetic tension. However, in an open field configuration, which we assume, this force is more than compensated by the second term, which represents an outward tension. Since this second term cannot be estimated within the context of a spherical model, we simply ignore the last two terms, representing tension. Numerical simulations (cf. Fiedler \& Mouschovias, 1992; Lizano \& Shu, 1989) show that during the quasistatic phase the field does not develop a strong curvature. Therefore, our neglect of tension should be accurate within a factor $\sim 2$.

To repeat, then, we ignore the last two terms in equation (41) and keep the first term, representing pressure. We eliminate the angular dependence of $B_{t}^{2}$ by using its angular average (cf. Chiueh \& Chou 1994); and, for simplicity, we shall use the notation $B$ instead of $\left\langle B_{t}^{2}\right\rangle^{1 / 2}$, where \langle\rangle stand for the angular average.

Finally, at the typical densities of dense molecular clouds the ionization fraction is very small, and therefore, to a good degree of approximation, we have that

$$
\rho_{i}\left(\frac{\partial v_{i}}{\partial t}+v_{i} \frac{\partial v_{i}}{\partial r}\right) \approx 0
$$

and

$$
\frac{G M \rho_{i}}{r^{2}} \approx 0
$$

With these assumptions of negligible thermal pressure and of spherical symmetry, and neglecting the inertia of the ions, the momentum equations for the neutral and ion fluids and the mass conservation equation for the neutrals take the form

$$
\begin{aligned}
\rho_{n} \frac{D v_{n}}{D t} & =\rho_{i} \rho_{n} \frac{\langle\sigma v\rangle_{n i}}{m_{i}} v_{D}-\frac{G M \rho_{n}}{r^{2}} \\
0 & =-\rho_{i} \rho_{n} \frac{\langle\sigma v\rangle_{n i}}{m_{i}} v_{D}-\frac{1}{8 \pi} \frac{\partial B^{2}}{\partial r}
\end{aligned}
$$

and

$$
\frac{D \rho_{n}}{D t}=-\frac{\rho_{n}}{r^{2}} \frac{\partial\left(r^{2} v_{n}\right)}{\partial r}
$$


where

$$
\frac{D}{D t} \equiv \frac{\partial}{\partial t}+v_{n} \frac{\partial}{\partial r}
$$

indicates a substantial derivative; $v_{D} \equiv v_{i}-v_{n}$ is the ion-neutral drift velocity; $\langle\sigma v\rangle_{n i}$ is the average collision rate between neutrals and ions, with the value $1.7 \times 10^{-9} \mathrm{~cm}^{-3} \mathrm{~s}^{-1}$ for $\mathrm{HCO}^{+}-\mathrm{H}_{2}$ collisons (McDaniel \& Mason, 1973); and all the other symbols have their usual meanings, with the subscripts $i$ and $n$ standing, respectively, for ion and neutral quantities. In writing equations (77) and (8), we have neglected the neutral mass in comparison with the ion mass, $m_{n} \ll m_{i}$. We define a new constant $k_{n i}$ by

$$
k_{n i} \equiv K_{i} \frac{\langle\sigma v\rangle_{n i}}{m_{n}^{1 / 2}}=8.2 \times 10^{-3} \mathrm{~cm}^{3 / 2} \mathrm{~s}^{-1} \mathrm{~g}^{-1 / 2},
$$

where $m_{n}$ and $m_{\mathrm{H}}$ are, respectively, the neutral's and proton's mass, and where we have set $m_{n}=2.33 m_{\mathrm{H}}$.

At this point it is important to make contact with previous work by other authors. We choose to focus on the solutions by Mouschovias and collaborators (see references in $\S 1$ ) because our approach is closest to theirs. Their solutions depend on three dimensionless parameters, one of which is (Fiedler \& Mouschovias, 1992)

$$
\nu_{\mathrm{ff}}=\left(\frac{8}{3 \pi^{2}}\right)^{1 / 2} \frac{t_{\mathrm{ff}, 0}}{t_{n i, 0}}
$$

where

$$
t_{\mathrm{ff}, 0}=\left(\frac{3 \pi}{32 G \rho_{n, 0}}\right)^{1 / 2}
$$

is the free-fall time for their initial reference state with uniform neutral density $\rho_{n, 0}$, and where

$$
t_{n i, 0}=\frac{1}{n_{i, 0}\langle\sigma v\rangle_{n i}}
$$

is the neutral-ion collision time for the initial reference state (note that $\nu_{\mathrm{ff}}$ depends only on universal constants and the microphysics of the problem, and that we have again assumed $\left.m_{n} \ll m_{i}\right)$. Our parameter $k_{n i}$ is directly related to $\nu_{\text {ff }}$ through

$$
k_{n i}=3.54 G^{1 / 2} \nu_{\mathrm{ff}} .
$$

Using $k_{n i}$ and equation (11), the system of eqs. (7) and (8) take the form

$$
\begin{aligned}
\rho_{n} \frac{D v_{n}}{D t} & =k_{n i} \rho_{n}^{3 / 2} v_{D}-\frac{G M \rho_{n}}{r^{2}} \\
0 & =-k_{n i} \rho_{n}^{3 / 2} v_{D}-\frac{1}{8 \pi} \frac{\partial B^{2}}{\partial r}
\end{aligned}
$$

In principle, one has to add to eqs. (9), (16), and (17) the induction equation to solve self-consistently for the magnetic field. We postpone our discussion of the induction equation until 
we consider other approximations that apply to the solution of eqs. (9), (16), and (17); it turns out that, to the same order of approximation for which our solution of eqs. (9), (16), and (17) holds, the induction equation is automatically satisfied to sufficient accuracy.

Our fundamental approximations to solve eqs. (9), (16), and (17) are two. First, we assume that the evolution is quasi-static, and set

$$
\frac{D v_{n}}{D t} \approx 0
$$

Second, we assume that the ions are effectively static, so that their velocity is small compared even with that of the neutrals:

$$
v_{i} \ll v_{n}
$$

so that

$$
v_{D} \approx-v_{n}
$$

These approxmations require comment. Recall that we are interested in the case where only the magnetic field supports the cloud against gravity. Because only the ions are directly coupled to the field, the neutrals are subject to the magnetic forces only through collisions with the ions, and there is a net slip of the neutral material past the magnetic field lines (Mestel \& Spitzer, 1956). If this collisional coupling is effective, the drift velocity $v_{D}$ will be small. There are two ways in which this can occur: On the one hand, when $v_{i} \sim v_{n}$, the drift velocity is small, but the ions are essentially comoving with the neutrals; in other words, the cloud is not magnetically supported and it undergoes dynamical collapse (Mestel \& Spitzer, 1956; note that when $v_{i}=v_{n}$ the first term on the rhs of equation [16] vanishes, and the material is in free-fall). On the other hand, $v_{D}$ is small also when $v_{i} \ll v_{n}$, and $v_{n}$ itself is small. In this case, the ions - and the magnetic field lines - are essentially stationary, and the neutral material slowly drifts by them. Thus, provided that $v_{n}$ is much smaller than the local free-fall velocity, the coupling between the ions and the neutrals is good, and the magnetic field is effective in supporting the cloud on timescales of order the free-fall time, but on a longer timescale the cloud is in a state of quasistatic contraction (e.g., Lizano \& Shu 1989; Mouschovias, 1991). This is the case we consider here: quasi-static contraction of the neutrals through essentially static ions.

Using eqs. (18) and (20), equation (16) becomes

$$
v_{n}=-\frac{G M}{k_{n i} r^{2} \rho_{n}^{1 / 2}} .
$$

Substitution into equation (9) then gives

$$
\frac{D \rho_{n}}{D t}=\frac{G}{k_{n i}} \frac{\rho_{n}}{r^{2}} \frac{\partial}{\partial r}\left(\frac{M}{\rho_{n}^{1 / 2}}\right) .
$$

Lagrangian coordinates are better suited for the solution of equation (22). By using the transformation

$$
\frac{\partial r}{\partial M}=\frac{1}{4 \pi r^{2} \rho_{n}}
$$


on equation (22), we finally get our fundamental equation:

$$
\frac{D \rho_{n}}{D t}=\frac{4 \pi G}{k_{n i}} \rho_{n}^{2} \frac{\partial}{\partial M}\left(\frac{M}{\rho_{n}^{1 / 2}}\right) .
$$

Note that equation (24) is a self-contained equation for $\rho_{n}(t, M)$; once a solution is found, $v_{n}$ and $r$ can be found from eqs. (21) and (23), respectively, while $B$ is obtained by quadrature of

$$
\frac{\partial B^{2}}{\partial M}=-\frac{2 G M}{r^{4}}
$$

which follows from eqs. (16) and (17) in the limit where eqs. (5), (6), and (18) are valid.

\subsection{Self-Consistency and the Induction Equation}

One can check, a posteriori, the validity of the quasi-static approximation (eq. [18) in the following way. For a given solution, evaluate the Lagrangian time derivative of $v_{n}$. Then, in terms of the parameter $\alpha$ defined by

$$
\frac{D v_{n}}{D t} \equiv-\alpha \frac{G M}{r^{2}(M)}
$$

the quasi-static approximation is valid as long as $\alpha \ll 1$.

Our second approximation in solving eqs. (9), (16), and (17) is given by equation (19), i.e., that the motion of the ions is negligible compared to that of the neutral material. The magnitude of $v_{i}$ is critical to the solution of the induction equation,

$$
\frac{\partial \boldsymbol{B}}{\partial t}=\nabla \times\left(\boldsymbol{v}_{\boldsymbol{i}} \times \boldsymbol{B}\right) .
$$

In the limit where $v_{i}=0$ identically, the magnetic field is constant in time, and the neutrals simply move across the field lines, which are fixed in space. In this case, the spatial gradient of the field is given by the condition of hydrostatic equilibrium, equation (25), and the induction equation is automatically satisfied. This simple situation still applies for finite $v_{i}$ as long as $v_{i} \ll v_{n}$. To quantify matters, we rewrite the induction equation heuristically as

$$
\frac{\partial \Phi}{\partial t}+v_{i} \frac{\partial \Phi}{\partial r}=0,
$$

where $\Phi$ is the magnetic flux, defined by

$$
\frac{\partial \Phi}{\partial r}=2 \pi B r .
$$

Equation (28) can be rewritten as

$$
\frac{\partial \Phi}{\partial t}=-2 \pi \frac{v_{i}}{v_{n}} v_{n} B r .
$$


Let $L$ be a characteristic length of the problem, and $t_{n}$ a characteristic time for the motion of the neutrals; then

$$
v_{n} \sim \frac{L}{t_{n}}
$$

and to within order of magnitude equation (30) reads

$$
\frac{\partial \Phi}{\partial t} \sim \frac{v_{i}}{v_{n}} \frac{B L^{2}}{t_{n}} \sim \frac{v_{i}}{v_{n}} \frac{\Phi}{t_{n}} .
$$

Equation (32) shows that the characteristic time for the magnetic flux to change is a factor $v_{n} / v_{i}$ longer than the characteristic time for the motion of the neutrals. Therefore, as long as

$v_{i} / v_{n} \ll 1$ the induction equation need not be solved separately, and the strength of the magnetic field is given by the condition of hydrostatic equilibrium. This means that the magnetic and hydrodynamic parts of the problem decouple, resulting in an enormous simplification.

The validity of the condition $v_{i} / v_{n} \ll 1$ can be checked a posteriori by solving for $v_{i}$ from equation (28),

$$
v_{i}=-\frac{\partial \Phi / \partial t}{\partial \Phi / \partial r}
$$

once $B$ and $\Phi$ are found, respectively, from eqs. (25) and (29).

Finally, the assumption that the thermal stresses are negligible compared to the magnetic stresses can be checked a posteriori by evaluating a modified form of the parameter $\beta$ in equation (2):

$$
\beta^{\prime}=8 \pi k_{\mathrm{B}} T \frac{\partial n / \partial r}{\partial B^{2} / \partial r}
$$

\subsection{Dimensionless Expressions}

It is convenient to cast all the equations in dimensionless form. Let

$$
\begin{aligned}
\rho_{n}(t, M) & =\rho_{\mathrm{c}, 0} \hat{\rho}(\tau, m), \\
r(t, M) & =R_{0} \hat{r}(\tau, m),
\end{aligned}
$$

and

$$
B(t, M)=B_{0} b(\tau, m),
$$

where $\rho_{\mathrm{c}, 0}, R_{0}$, and $B_{0}$ are, respectively, the initial central density, the initial radius of the cloud, and the magnetic field at the edge of the cloud, and where we have introduced a dimensionless time $\tau$ and a dimensionless mass $m$ :

$$
\begin{aligned}
t & =t_{\mathrm{AD}} \tau, \\
M & =M_{0} m .
\end{aligned}
$$


Here $M_{0}$ is the total mass of the cloud, and $t_{\mathrm{AD}}$ is a characteristic ambipolar diffusion timescale,

$$
t_{\mathrm{AD}} \equiv \frac{k_{n i}}{4 \pi G \rho_{c, 0}^{1 / 2}}=4.96 \times 10^{6}\left(\frac{n_{c, 0}}{10^{3} \mathrm{~cm}^{-3}}\right)^{-1 / 2} \mathrm{yr},
$$

where $n_{c, 0}$ is the initial number density at the center of the cloud. Note that the ratio $t_{\mathrm{AD}} / t_{\mathrm{ff}}$, where $t_{\mathrm{ff}}$ is the characteristic free-fall time for the inital state, is given by

$$
\frac{t_{\mathrm{AD}}}{t_{\mathrm{ff}}}=0.147 \frac{k_{n i}}{G^{1 / 2}}=4.7
$$

or, in terms of the quantity $\nu_{\mathrm{ff}}$ used by Mouschovias and collaborators,

$$
\frac{t_{\mathrm{AD}}}{t_{\mathrm{ff}}}=\left(\frac{8}{3 \pi^{2}}\right)^{1 / 2} \nu_{\mathrm{ff}}
$$

Let us define the parameter $\epsilon$, which is inversely related to the initial degree of concentration of the cloud:

$$
\epsilon \equiv \frac{M_{0}}{\frac{4 \pi}{3} R_{0}^{3} \rho_{\mathrm{c}, 0}}=\frac{\langle\rho\rangle_{0}}{\rho_{\mathrm{c}, 0}} .
$$

Also, let $\hat{v}_{i}$ and $\hat{v}_{n}$ be, respectively, the dimensionless ion and neutral velocities, defined to be positive,

$$
v_{n} \equiv-v_{n, 0} \hat{v}_{n}
$$

and

$$
v_{i} \equiv-v_{n, 0} \hat{v}_{i}
$$

Here,

$$
v_{n, 0} \equiv \frac{G M_{0}}{k_{n i} R_{0}^{2} \rho_{\mathrm{c}, 0}{ }^{1 / 2}}=\epsilon \frac{R_{0}}{3 t_{\mathrm{AD}}}
$$

is a characteristic velocity of the neutrals. Note that the initial velocity of the neutrals at the edge of the cloud is $v_{n}=v_{n, 0}\left[\rho_{c, 0} / \rho_{0}\left(R_{0}\right)\right]^{1 / 2} \geq 1$.

To characterize the magnetic field, we define a parameter $\delta$ as the initial ratio of gravitational to magnetic pressure at the edge of the cloud:

$$
\delta \equiv \frac{2 G M_{0}^{2}}{R_{0}^{4} B_{0}^{2}}
$$

The magnetic field can also be characterized in terms of the magnetic critical mass $M_{\Phi}$, defined by

$$
M_{\Phi}=\frac{c_{\Phi}}{G^{1 / 2}} \Phi
$$

where $c_{\Phi}$ is a numerical constant that depends on the distribution of the mass to flux ratio in the cloud; for a uniform spherical cloud with a constant magnetic field, $c_{\Phi} \simeq 0.12$ (Tomisaka et al. 1988b). Clouds with $M>M_{\Phi}$ (magnetically supercritical) are dynamically unstable and will 
collapse even if the magnetic field is perfectly coupled to the neutral material. In terms of the characteristic value for the initial magnetic critical mass $M_{\Phi 0} \equiv c_{\Phi} \pi R_{0}^{2} B_{0} / G^{1 / 2}$, we have

$$
\frac{M_{\Phi 0}}{M_{0}}=\frac{\pi c_{\Phi} \sqrt{ } 2}{\delta^{1 / 2}}=\frac{0.53}{\delta^{1 / 2}} .
$$

We shall focus on clouds that have small values of $\delta$, corresponding to magnetically subcritical clouds $\left(M_{\Phi 0} / M_{0}>1\right)$ in which magnetic forces are initially stronger than gravitational forces.

Using the definitions in eqs.(35)-(47), our basic equations (23), (24), and (25) take the form

$$
\begin{aligned}
\frac{D \hat{\rho}}{D \tau} & =\hat{\rho}^{2} \frac{\partial}{\partial m}\left(\frac{m}{\hat{\rho}^{1 / 2}}\right), \\
\frac{\partial \hat{r}^{3}}{\partial m} & =\frac{\epsilon}{\hat{\rho}} \\
\frac{\partial b^{2}}{\partial m} & =-\delta \frac{m}{\hat{r}^{4}}
\end{aligned}
$$

and equation (21) now reads

$$
\hat{v}_{n}=\frac{m}{\hat{r}^{2} \hat{\rho}^{1 / 2}} .
$$

The solution of equation (50) requires the specification of an initial condition $\hat{\rho}_{0} \equiv \hat{\rho}(\tau=0, m)$. In addition, equations (51) and (52) are subject to the boundary conditions

$$
\begin{aligned}
\hat{r}(\tau=0, m=1) & =1, \\
\left.\frac{\partial \hat{r}}{\partial \tau}\right|_{m=0} & =0,
\end{aligned}
$$

and

$$
b(\tau, m=1)=1,
$$

respectively. The boundary conditions in eqs. (54)-(55) are obvious, while the boundary condition given by equation (56) means that the magnetic field at the cloud's edge is constant. This boundary condition is appropriate for a dense cloud surrounded by a low density medium threaded by a field $B_{0}$.

As an a posteriori check, the assumption of quasi-static motion can be quantified with the help of the quantity $\alpha$ defined in equation (26)

$$
\begin{aligned}
\alpha & =\frac{4 \pi G}{k_{n i}{ }^{2}} \frac{\hat{r}^{2}}{m} \frac{D \hat{v}_{n}}{D \tau} \\
& =1.2 \times 10^{-2} \frac{\hat{r}^{2}}{m} \frac{D \hat{v}_{n}}{D \tau},
\end{aligned}
$$

where we used the value of $k_{n i}$ in equation (11); the ratio $v_{i} / v_{n}$ is given by

$$
\frac{v_{i}}{v_{n}}=\frac{3}{\epsilon} \frac{1}{\hat{v}_{n}} \frac{\partial \phi / \partial \tau}{\partial \phi / \partial \hat{r}}
$$


where $\phi$ is the dimensionless magnetic flux defined by

$$
\Phi=\pi B_{0} R_{0}^{2} \phi
$$

and, finally, by defining a central value of the parameter $\beta$ in equation (2), we can quantify the role of thermal stresses with

$$
\begin{aligned}
\beta^{\prime} & =\frac{8 \pi k_{\mathrm{B}} n_{\mathrm{c}, 0} T}{B_{0}^{2}} \frac{\partial \hat{\rho} / \partial m}{\partial b^{2} / \partial m} \\
& \equiv \beta_{0} \frac{\partial \hat{\rho} / \partial m}{\partial b^{2} / \partial m}
\end{aligned}
$$

where $n_{c, 0}$ is the initial number density at the center of the cloud.

To summarize, by putting our problem in dimensionless form, we have reduced the dependence of the solution to only two free parameters, $\epsilon$ and $\delta$, together with a specification of the initial mass distribution in the cloud. The parameter $\epsilon$ is the inverse of the initial degree of concentration of the cloud (eq. [43]), and the evolution of the hydrodynamical variables $\hat{r}, \hat{v}$, and $\hat{\rho}$ depends only on this parameter. The second free parameter, $\delta$ (eq. [47]), is the initial ratio of gravitational to magnetic pressure at the edge of the cloud, and parameterizes the initial mass-to-flux ratio of the cloud (eq. [49]). The evolution of the magnetic field and the ion velocity depends on $\delta$ and $\epsilon$. Also, we will show below (eq. [83]) that the value of $\delta$ determines the regime where our static-ion approximation is valid. To convert our dimensionless solution into physical units, it is necessary to specify the cloud mass $M_{0}$, the initial cloud radius $R_{0}$, the magnetic field at the edge of the cloud $B_{0}$, and the ambipolar diffusion time $t_{\mathrm{AD}}$ (eq. [40]) or the initial density at the center of the cloud $\rho_{c, 0}$.

\subsection{A General Solution}

The key to the solution of the system of equations (50)-(53) is the solution of equation (50). This can easily be achieved by defining the new dependent variable

$$
\chi \equiv \frac{m}{\hat{\rho}^{1 / 2}}
$$

and the new independent variable

$$
\xi \equiv \frac{1}{m} .
$$

Using these transformations, equation (50) takes the standard form

$$
a(\chi) \frac{\partial \chi}{\partial \xi}+\frac{\partial \chi}{\partial \tau}=0
$$

where

$$
a(\chi)=-\frac{1}{2 \chi} .
$$


Equation (63) is a one-dimensional conservation law: if $\chi_{0}(\xi) \equiv \chi(\tau=0, \xi)$ is the initial condition for $\chi$, then the solution of equation (63) is given by

$$
\chi(\tau, \xi)=\chi_{0}[\xi-a(\chi) \tau]
$$

Two things are remarkable about the solution given by equation (65). First, it represents an implicit equation for $\chi$, and one hopes that for certain forms of the initial condition $\chi_{0}(\xi)$ this equation can be solved algebraically. Second, note that the solution for $\chi$ has the form of a wave with velocity $a(\chi)$, and therefore there exists the possibility of wave steepening and shock formation, i.e., the formation of a singularity.

With $a(\chi)$ given by equation (64), the solution in equation (65) becomes

$$
\chi(\tau, \xi)=\chi_{0}(\psi \xi)
$$

where

$$
\psi \equiv 1+\frac{\tau}{2} \hat{\rho}^{1 / 2}
$$

Because $\chi=\left(\hat{\rho}^{1 / 2} \xi\right)^{-1}$, we have the solution for the density in terms of its initial distribution:

$$
\hat{\rho}(\tau, \xi)=\psi^{2} \hat{\rho}_{0}(\psi \xi)
$$

To proceed, we must select a specific form for the inital density distribution $\hat{\rho}_{0}(\xi)$. A form that roughly approximates models for molecular cores is

$$
\hat{\rho}_{0}=\frac{m_{c}^{p}}{\left(m_{\mathrm{c}}+m\right)^{p}}=\left(1+\frac{1}{m_{c} \xi}\right)^{-p}
$$

with $m_{\mathrm{c}}$ a constant. We shall concentrate on two cases: $p=2$, which approximates the density profile of a non-singular isothermal cloud, and $p=0$, which provides useful insights into the evolution of the central region. First, however, we consider some features of the general case.

\section{The Case of Arbitrary $p$}

Because

$$
m=M_{0}^{-1} \int_{0}^{r} 4 \pi r^{2} \rho d r
$$

equation (69) is an integral equation for $\hat{\rho}_{0}(\hat{r})$, the initial density as a function of radius. This equation is readily solved to give

$$
\hat{\rho}_{0}=\left[1+\frac{(p+1) \hat{r}^{3}}{\epsilon m_{c}}\right]^{-\frac{p}{p+1}}
$$


Inserting this result back into equation (69) gives

$$
\frac{m}{m_{\mathrm{c}}}=\left[1+\frac{(p+1) \hat{r}^{3}}{\epsilon m_{c}}\right]^{\frac{1}{p+1}}-1 \quad(\tau=0),
$$

and because, initially, $m=1$ at $\hat{r}=1$, this result provides the relation between $\epsilon$ and $m_{\mathrm{c}}$ for our model,

$$
\epsilon=\frac{p+1}{m_{\mathrm{c}}\left[\left(1+\frac{1}{m_{c}}\right)^{p+1}-1\right]} .
$$

We now evaluate the solution given by equation (68) for the initial density distribution we have adopted,

$$
\hat{\rho}=\psi^{2}\left(1+\frac{m}{m_{c} \psi}\right)^{-p}
$$

Using equation (67) for $\psi$, we obtain the general solution

$$
\frac{m}{m_{c}}=\left(1+\frac{\tau}{2} \hat{\rho}^{1 / 2}\right)\left[\left(\frac{1}{\hat{\rho}^{1 / 2}}+\frac{\tau}{2}\right)^{2 / p}-1\right] .
$$

We obtain the central density $\hat{\rho}_{c}$ by setting $m=0$,

$$
\hat{\rho}_{c}=\frac{4}{(2-\tau)^{2}}
$$

Thus, the solution becomes singular at $\tau=2$. Near the center of the cloud, the density becomes very large as $\tau \rightarrow 2$. We can expand equation (75) as this limit is approached and find

$$
\frac{m}{m_{c}} \rightarrow \frac{2}{p}\left[1+\frac{2+p}{2 p \hat{\rho}^{1 / 2}}\right] .
$$

We conclude that at $\tau=2$ a core of mass $(2 / p) m_{c}$ collapses to a singularity. This behavior is an artifact of our neglect of thermal pressure. Had we included thermal pressure, the singularity would have been avoided, and instead a high density object - a protostar - would have formed at $\tau \approx 2$.

One other feature of the solution at $\tau=2$ is worth noting. Equation (77) shows that the mass just outside the singularity scales as $\hat{\rho}^{-1 / 2}$. Assuming a power law for for $\hat{\rho}(\hat{r})$ there, we find $\hat{\rho}(\tau=2, \hat{r}) \propto \hat{r}^{-2}$ : at the instant the singularity forms, the density just outside has the same radial dependence as a singular isothermal sphere. However, this gas is not static.

The conditions for the solution found here to be valid are more easily discussed for specific models, so we now consider the collapse of a homogeneous cloud. 


\section{The Homogeneous Cloud}

The case of an initially homogeneous cloud $(p=0, \epsilon=1)$ is of particular interest because a complete solution can be obtained analytically, and because it gives some insights into how the collapse of the inner core of a stratified cloud takes place. Here we have $\hat{\rho}_{0}=1$ and $\hat{\rho}=\psi^{2}$, so that the complete solution is given by

$$
\begin{aligned}
\hat{\rho} & =\frac{4}{(2-\tau)^{2}}, \\
\hat{r}^{3} & =\frac{1}{4} m(2-\tau)^{2}, \\
\hat{v}_{n} & =\frac{(2 m)^{1 / 3}}{(2-\tau)^{1 / 3}}, \\
b & =\left[1+\frac{3}{2} \frac{2^{8 / 3} \delta}{(2-\tau)^{8 / 3}}\left(1-m^{2 / 3}\right)\right]^{1 / 2},
\end{aligned}
$$

and

$$
\begin{aligned}
\phi= & \frac{4}{9} \frac{1}{\delta} \frac{(2-\tau)^{4}}{16}\left\{\left[1+\frac{3}{2} \frac{2^{8 / 3} \delta}{(2-\tau)^{8 / 3}}\right]^{3 / 2}-\right. \\
& {\left.\left[1+\frac{3}{2} \frac{2^{8 / 3} \delta}{(2-\tau)^{8 / 3}}\left(1-m^{2 / 3}\right)\right]^{3 / 2}\right\} . }
\end{aligned}
$$

There are several things that are worth noting. First, $\hat{\rho}$ remains uniform even at late times, just as in the collapse of a non-magnetic spherical cloud (Spitzer, 1978). The collapse is homologous, with $v \propto r$, since the acceleration rises linearly with $r$ for a homogeneous cloud. Figure 1 is a plot of $\hat{r}$ as a function of $\tau$ for various values of $m$, in a cloud with $\delta=4 \times 10^{-3}$, which is appropriate for a cloud of $M_{0}=5 \mathrm{M}_{\odot}, R_{0}=0.45 \mathrm{pc}$, and $B_{0}=30 \mu \mathrm{G}$; such a cloud has $M_{\Phi 0} / M_{0}=8.3$, which is quite magnetically subcritical. Next, note that the expressions for the magnetic field and the flux introduce a characteristic time, $\tau_{\mathrm{cr}}$, which we choose to define as

$$
2-\tau_{\mathrm{cr}}=2(3 \delta)^{3 / 8} \text {. }
$$

For the case at hand, $\tau_{\mathrm{cr}}=1.62$.

Because the outer parts of the cloud accelerate first, one is tempted to conclude that it is the cloud as a whole that first becomes gravitationally unstable, rather than the central region. This hypothesis can be tested by evaluating $M_{\Phi} / M$ as a function of time and mass. For flattened clouds, it is customary to evaluate this ratio for a flux tube. In our spherical geometry, however, we evaluate $M(r)$, the mass inside a sphere of radius $r$, and compare this with the flux $\Phi(r)$. Had we included the pressure that makes the cloud spherical, the condition for gravitational instability would have been that $M(r)$ exceed both the Jeans mass and the magnetic critical mass $M_{\Phi}$. 
Having $M(r)>M_{\Phi}(r)$ is thus a necessary condition for gravitational instability. Figures 2 a and $2 \mathrm{~b}$ show

$$
\frac{M_{\Phi}}{M}=\frac{M_{\Phi 0}}{M_{0}}\left(\frac{\phi}{m}\right)=\frac{0.53}{\delta^{1 / 2}}\left(\frac{\phi}{m}\right)
$$

where we have made use of equation (49). Note that at $\tau=0$ the cloud is magnetically subcritical everywhere $\left(M_{\Phi} / M>1\right)$, and that the outer edge of the cloud first becomes unstable at $\tau \simeq 1.62=\tau_{\mathrm{cr}}$. More precisely, at $\tau=\tau_{\mathrm{cr}}$, we have $\phi(m=1)=1.93 \delta^{1 / 2}$, so that $M_{\Phi} / M_{0}=1.03$, independent of the value of $\delta$. Thus, $\tau_{\text {cr }}$ is very nearly equal to the time at which the cloud first becomes supercritical.

These results show that in a homogeneous, cold, and magnetized cloud the collapse due to ambipolar diffusion proceeds from outside-in the The inner regions of the cloud are gravitationally stable, and collapse only because of the weight of the overlying material. By contrast, the collapse of a non-magnetized singular isothermal sphere occurs inside-out (Shu 1977): such a sphere is unstable at all points, but the collapse begins at the origin, where the free-fall time is the shortest.

Finally, we determine $\phi_{\text {core }}$, the residual flux trapped in the cloud when it collapses to a singularity at $\tau=2$. Taking the limit of $\phi(m=1)$ as $\tau \rightarrow 2$ in equation (82), we find $\phi_{\text {core }}=(2 \delta / 3)^{1 / 2}$. As a result, we have

$$
\frac{M_{\Phi, \text { core }}}{M_{0}}=\left(\frac{M_{\Phi 0}}{M_{0}}\right) \phi_{\text {core }}=0.43 .
$$

Our model thus shows that the trapped flux is sufficiently small that the collapsed core is magnetically supercritical, as expected.

Are these results consistent with our assumptions? The validity of the quasi-static approximation can be readily assessed with the help of the parameter $\alpha$ - the acceleration of the neutrals in units of the local gravitational acceleration (eq. [26]) - and the expressions in eqs. (79) and (80), which yields

$$
\frac{\hat{r}^{2}}{m} \frac{D \hat{v}_{n}}{D \tau}=\frac{1}{6}
$$

Therefore

$$
\alpha=2.1 \times 10^{-3},
$$

and the approximation of quasi-static evolution is very good, at least so long as our static ion approximation holds.

Next, the assumption of negligible ion velocity compared to the neutral velocity has to be checked. Figure 3 is a plot of $v_{i} / v_{n}$ as a function of the Lagrangian coordinate $M$ (in units of

\footnotetext{
${ }^{4}$ It is interesting to note that this outside-in collapse has been found by Goldreich \& Weber (1980) in a completely different context. They studied the core collapse in supernova progenitors with an equation of state $P \propto \rho^{4 / 3}$. In our case, this equation of state becomes appropriate once the magnetic field has been amplified considerably, so that $b \gg 1$ (see eqs. 78 and 81]). Goldreich \& Weber's results show that the dynamical evolution of the core is homologous, with the outside of the core accelerating first.
} 
the cloud's mass $M_{0}$ ) for four different times. It can be seen that up to $t / t_{\mathrm{AD}}=1.62$-i.e., at $\tau_{\text {cr }}$, when the cloud as a whole becomes magnetically supercritical $-v_{i} / v_{n} \lesssim 0.3$; therefore our assumption of negligible $v_{i}$ is justified a posteriori, up to the point where the cloud becomes fully supercritical. Once the cloud is supercritical, $v_{i}$ begins to approach $v_{n}$, and our solution breaks down. This accounts for the late-time behavior of $M_{\Phi} / M_{0}$ seen in Figure 2b: after $\tau=1.62$ this ratio does not change much, as expected when the magnetic flux starts to become frozen onto the neutrals. We conclude that the static ion approximation breaks down at about the same time the cloud becomes magnetically supercritical; this ultimately leads to the breakdown of the quasi-static approximation for the neutrals.

We can analytically verify that the static ion approximation breaks down when the cloud becomes supercritical. Note that the maximum value of $v_{i} / v_{n}$ occurs at the center of the cloud. According to equation (58), $v_{i} / v_{n}$ depends on the ratio of $\partial \phi / \partial \tau$ to $\partial \phi / \partial \hat{r}$. Near the center of the cloud, we have $\phi \simeq \pi \hat{r}^{2} b\left(m \ll m_{c}\right)$. Evaluating the partial derivatives, we find

$$
\left(\frac{v_{i}}{v_{n}}\right)_{m=0}=\left[1+2\left(\frac{2-\tau}{2-\tau_{\mathrm{cr}}}\right)^{8 / 3}\right]^{-1} .
$$

This result confirms that the ions are approximately static prior to $\tau_{\mathrm{cr}}$, when $v_{i} / v_{n}=1 / 3$. Note that this estimate indicates that the ions and neutrals become comoving as $\tau \rightarrow 2$, as they do in reality.

A remarkable feature of the solution discussed in this section is that it is completely independent of one of the basic parameters of the problem, the ratio of the ambipolar diffusion time to the free fall time $\left(\nu_{\mathrm{ff}} \propto t_{\mathrm{AD}} / t_{\mathrm{ff}}\right)$. The reason for this is now clear: our solution is valid only during the magnetically subcritical, quasi-static stage of evolution, when the timescale is determined by $t_{\mathrm{AD}}$ alone. The solution sets the initial conditions for the subsequent supercritical, dynamical stage of evolution, when the timescale is set by $t_{\mathrm{ff}}$.

\section{A Stratified, Non-Singular Cloud}

We now consider a more realistic case, namely, that of a stratified, non-singular cloud. For an initial density profile of the form

$$
\hat{\rho}(\tau=0, m)=\frac{m_{\mathrm{c}}^{2}}{\left(m_{\mathrm{c}}+m\right)^{2}}
$$

(i.e., choosing $p=2$ in eq. [69]) equation (75) can be inverted algebraically, and we obtain

$$
\hat{\rho}=\frac{4}{\left[1+\frac{m}{m_{c}}-\tau+\sqrt{\left(1+\frac{m}{m_{c}}\right)^{2}-2 \tau \frac{m}{m_{c}}}\right]^{2}} .
$$

Recall that this case is a good approximation to a stable, non-singular, isothermal sphere, which is relevant to the study of real clouds. 
Armed with the solution in equation (90), we can solve for $\hat{r}(\tau, m)$ using equation (51) and the boundary conditions in equations (54)-(55):

$$
\begin{aligned}
\hat{r}^{3}(\tau, m)=\frac{m_{\mathrm{c}} \epsilon}{4}\left\{\frac{2}{3}\right. & {\left[\left(1+\frac{m}{m_{c}}\right)^{2}-2\left(\frac{m}{m_{c}}\right) \tau\right]^{3 / 2}+\frac{2}{3}\left(1+\frac{m}{m_{c}}\right)^{3} } \\
& \left.+\left(\frac{m}{m_{c}}\right) \tau^{2}-2\left(\frac{m}{m_{c}}\right)\left(1+\frac{m}{m_{c}}\right) \tau-\frac{4}{3}\right\} .
\end{aligned}
$$

What is the nature of the solution given by equations (90) and (91)? Recall that at the time $\tau=2$, a core of mass $M_{c}=m_{\mathrm{c}} M_{0}$ collapses into the origin, leaving behind an envelope with mass $\left(1-m_{\mathrm{c}}\right) M_{0}$. Evaluating $\hat{\rho}$ and $\hat{r}^{3}$ at $\tau=2$, they take the form

$$
\hat{\rho}(\tau=2, m)=4\left[\frac{m}{m_{\mathrm{c}}}-1+\left|1-\frac{m}{m_{\mathrm{c}}}\right|\right]^{-2}
$$

and

$$
\hat{r}^{3}(\tau=2, m)=\frac{m_{\mathrm{c}} \epsilon}{6}\left[\left(\frac{m}{m_{\mathrm{c}}}-1\right)^{3}+\left|\frac{m}{m_{\mathrm{c}}}-1\right|^{3}\right],
$$

respectively. Note that for $m \leq m_{\mathrm{c}}$ the expression between the square brackets in equation (93) vanishes, and $\hat{r}=0$ identically. We conclude that in a cloud that is initially everywhere magnetically subcritical, a core with a fraction $m_{\mathrm{c}}$ of the total cloud mass will, through ambipolar diffusion, eventually become supercritical, and collapse into the origin. The relationship between $m_{\mathrm{c}}$ and the initial degree of concentration of the cloud, $\epsilon$, given by equation (73) with $p=2$, is plotted in Figure 4. We find that $m_{\mathrm{c}}=1$ for $\epsilon=3 / 7$, and therefore clouds with $\epsilon \geq 3 / 7$ will collapse as a whole, leaving no envelope behind.

\subsection{Evolution Prior to Collapse}

Further insight into the nature of the solution can be gained by examining in detail the time evolution. Here we consider the evolution up to the formation of a singularity in the origin of a cloud with $\epsilon=0.1$ (corresponding to $m_{\mathrm{c}}=0.26$ ) and $\delta=4 \times 10^{-3}$ (or, equivalently, $M_{\Phi, 0} / M_{0}=8.4$ ). These values correspond to a cloud of mass $M_{0}=5 \mathrm{M}_{\odot}$, initial radius $R_{0}=0.45 \mathrm{pc}$, a magnetic field strength at the edge of the cloud $B_{0}=30 \mu \mathrm{G}$, and an initial particle density at the center of $n_{c, 0}=2.2 \times 10^{3} \mathrm{~cm}^{-3}$-yielding a characteristic ambipolar diffusion time $t_{\mathrm{AD}}=3.30 \mathrm{Myr}$.

\footnotetext{
${ }^{5}$ The boundary condition given by equation $(55)$ is strictly valid for $\tau<2$ because $\left(\tau=2, m \leq m_{c}\right)$ is a singular point of equation (51). However, the solution for $\hat{r}(\tau, m)$ given by equation (91) can still be used for $\tau>2$ and $m>m_{c}$. The reason is that the evolution of shells enclosing $m>m_{c}$ depends, by Newton's theorem, on $m$ only and not its distribution - whether pointlike or distributed over a finite volume. Therefore, the neutral's velocity $\hat{v}_{n}$ and the location of each shell $\hat{r}$ are smooth functions of $\tau$ across $\tau=2$ for $m>m_{c}$.
} 
Figure 5 is a plot of the Lagrangian position $r$, neutral velocity $v_{n}$, density $\rho$, and magnetic field strength $B$. Focusing on Figure $5 \mathrm{a}$, it is noteworthy that by $\tau=1.86$ the radius for $m=1$ is reduced by a factor $\sim 0.6$ from its initial value, while for $m \lesssim m_{\mathrm{c}}$ this change is by a factor of order $\sim 0.2$. Moreover, note how the change in radius is roughly the same for all $m$ in the region $m \lesssim m_{\mathrm{c}}$, i.e, the evolution of the core resembles that of a homogeneous cloud. This result is further illuminated by considering the behavior of $v_{n}$. Initially the absolute value of $v_{n}$ is largest at the outer edge of the cloud, but by $t / t_{\mathrm{AD}} \sim 1.6$ a maximum (in absolute value) starts to develop at $m \sim m_{\mathrm{c}}$, which becomes more accentuated as time goes on. In other words, the dynamical behavior of the core with $m \lesssim m_{\mathrm{c}}$ resembles that of a homogeneous cloud, and its collapse also proceeds from outside-in.

Finally, it is interesting to examine Figure $5 \mathrm{~b}$ and note that by $t / t_{\mathrm{AD}}=1.8$ the central density has increased by a factor $\sim 100$, while $B$ at the center has increased by only a factor $\lesssim 4$-as expected in a cloud whose evolution is dominated by ambipolar diffusion.

\subsection{Self-Consistency}

In this section we check the self-consistency of our results for our fiducial stratified cloud, namely, the validity of the quasi-static approximation, the neglect of thermal stresses, and the assumption that $v_{i} \ll v_{n}$, which allows us to neglect the induction equation.

\subsubsection{Quasi-static Evolution}

Figure 6 is a graph of $\alpha$, the acceleration of the neutrals in units of the local gravitational acceleration, as a function of $m$ and $\tau$. Note that, up to the time shown $(\tau=1.996), \alpha \ll 1$, i.e., the acceleration of the neutrals is a very small fraction of the local gravitational acceleration, and therefore the assumption of quasi-static evolution is very good. Also, note that for $m \lesssim 0.2, \alpha$ is roughly constant, as in a homogeneous cloud. However, just as in the case of the homogeneous cloud, it is the breakdown of the static ion approximation that occurs first, and that ultimately leads to dynamical evolution.

\subsubsection{Static-Ion Approximation}

A key assumption in our approach is that the ion velocity is much smaller than the neutral velocity, so that the hydrodynamic and magnetic problems are decoupled. This key assumption has to be verified a posteriori by computing the magnetic flux $\Phi$ (eq. [29]) and computing $v_{i}$ as prescribed by equation (33). The evolution of the magnetic flux as a function of mass and of position is shown in Fugure 7. Figure 8 is a plot of $v_{i} / v_{n}$ as a function of the dimensionless 
Lagrangian coordinate $M / M_{0}$ for five different times (in units of the characteristic ambipolar diffusion time $\left.t_{\mathrm{AD}}\right)$. Note that for $\tau \lesssim 1, v_{i} / v_{n} \ll 1$ obtains everywhere in the cloud, justifying our initial assumption. By $\tau=1.64, v_{i} / v_{n} \approx 0.35$ in the inner few percent (by mass) of the cloud. This result is similar to the one we obtained for the homogeneous cloud above. By plotting $M_{\Phi} / M$ (Figure 9), we see that a part of the cloud first becomes magnetically supercritical shortly before $\tau=1.64$. Hence, just as in the homogeneous case, the static ion approximation breaks down when the cloud becomes supercritical (although in this case only the core region is supercritical).

\subsubsection{The Validity of the $T=0$ Approximation}

One of our main simplifying assumptions is that thermal stresses are negligible compared to magnetic stresses. The validity of this approximation can be checked a posteriori by looking at the value of $\beta^{\prime}$, i.e, the ratio of the thermal to magnetic pressure gradients (eq. [34]). Figure 10 is a plot of $\beta^{\prime}$ as a function of $m$ and $\tau$ for our fiducial cloud. Note that $\beta^{\prime} \ll 1$ throughout the evolution; therefore, our assumption that thermal stresses can be neglected is justified.

At a given time (e.g., for $\left.t / t_{\mathrm{AD}}=0\right)$, our model predicts that the importance of thermal support would increase as one moves towards the center of the cloud until $m \lesssim m_{\mathrm{c}}$, where it starts to decrease. In a real cloud, one expects that, at the center, thermal pressure becomes important for a mass of order the Jean mass at the appropriate central density. Thus, one expects $\beta^{\prime}$ to increase towards the center of the cloud; whether at the center $\beta^{\prime} \sim 1$ depends on the particular magnetic configuration. Also, as ambipolar diffusion proceeds, the central density increases, and the mass - and size - of the region where thermal stresses are important decreases with time (recall that the Jeans mass $\propto\left(n_{c}\right)^{-1 / 2}$, where $n_{c}$ is the central density). In our case, the local maximum of $\beta^{\prime}$ obtains at a fixed value of $m$ - namely, $m=m_{\mathrm{c}}$ - because of our choice of initial conditions and our neglect of thermal stresses. However, this is not critical as long as we find that $\beta^{\prime} \ll 1$ throughout the cloud, because, as mentioned above, the Jeans mass decreases with increasing density. Thus, at worst, our calculation is in error in a region that becomes increasingly smaller with time.

As time increases, thermal pressure forces at the center may begin to dominate the magnetic stress due to the loss of magnetic flux. The answer to this question, again, depends on the particular magnetic configuration. In our case, we find that thermal stresses never become important during the quasistatic phase; this finding is in agreement with the results of Fiedler \& Mouschovias (1992), who find that even during the dynamical collapse of the core, magnetic stresses dominate over thermal pressure at least up to a density of $\sim 10^{9} \mathrm{~cm}^{-3}$. In our model, if there is no core to begin with-the constant density case- one never forms; (on the other hand, cores can form during the subsonic evolution of clouds supported by gas pressure - see Bodenheimer \& Sweigert, 1968). Once a core has formed, then we find that the subsequent contraction of the cloud results in a decrease in the relative importance of thermal stresses, as shown in Figure 10. 
Note that this predominance of magnetic support over thermal support does not contradict the observational evidence that the linewidths of dense cores are essentially thermal (see, e.g., Caselli \& Myers, 1995, and references therein). Recall that, in our picture, the support of the cloud is due to quasistatic magnetic fields - as opposed to magnetic turbulence - and that the drift velocities during the quasistatic phase are an order of magnitude below the speed of sound (see Figure 5a). Therefore, in our model, the main contribution to the line widths is thermal motion.

\subsection{Comparison with the Numerical Work of Fiedler \& Mouschovias}

Are our results relevant to the evolution of real clouds? To date, one can obtain an answer to this question only by comparison with state-of-the-art numerical calculations. Here we compare our results against those of Fiedler \& Mouschovias (1992) for their baseline model, Model 1. To effect the comparison, we need to specify five parameters: the time $t_{0}$ in Fiedler $\&$ Mouschovias's results that we identify with $\tau=0$, the corresponding central density $\rho_{\mathrm{c}, 0}$, the mass and initial radius of the cloud, $M_{0}$ and $R_{0}$ respectively, and the magnetic field at the edge of the cloud, $B_{0}$. The values of these last three parameters are fixed by Fiedler \& Mouschovias's choices, namely $M_{0}=45.5 \mathrm{M}_{\odot}, R_{0}=0.75 \mathrm{pc}$, and $B_{0}=30 \mu \mathrm{G}$; the values of $t_{0}$ and $\rho_{\mathrm{c}, 0}$ require further consideration.

Because Fiedler \& Mouschovias start their calculations with a homogeneous, uniformly magnetized cloud that relaxes to a quasi-static state, the only practical way to determine $t_{0}$ and $\rho_{\mathrm{c}, 0}$ is by fitting their results for the central density during the quasi-static phase with our expression for the central density (eq. [76] in dimensional form):

$$
\rho_{\mathrm{c}}(t)=4 \rho_{\mathrm{c}, 0}\left[2-\frac{4 \pi G \rho_{\mathrm{c}, 0}^{1 / 2}\left(t-t_{0}\right)}{k_{n i}}\right]^{-2} .
$$

Using the values adopted by Fielder \& Mouschovias in their Model 1 for the mean molecular weight, $K_{i}$, and $\langle\sigma v\rangle_{n i}$, we find that there is a continuum of equally good fits with $n_{\mathrm{c}, 0} \gtrsim 1.5 \times 10^{3} \mathrm{~cm}^{-3}$ or, equivalently, $\epsilon \lesssim 0.3$ - and $t_{0} \gtrsim 8.0 \mathrm{Myr}$. However, we show below that geometrical constraints narrow the range to $\epsilon \lesssim 0.1\left(n_{\mathrm{c}, 0} \gtrsim 4.5 \times 10^{3} \mathrm{~cm}^{-3}\right)$ and $t_{0} \gtrsim 10.0 \mathrm{Myr}$. We decide to adopt our fiducial value of $\epsilon=0.1$, and a very good fit obtains with $t_{0}=11.85 \mathrm{Myr}$. Given that $M_{0}$ and $R_{0}$ are fixed, this choice of $\epsilon$ translates into an initial central density $n_{\mathrm{c}, 0}=4.4 \times 10^{3} \mathrm{~cm}^{-3}$, and the characteristic ambipolar diffusion time for the cloud is $t_{\mathrm{AD}}=2.33 \mathrm{Myr}$. Finally, the choice $B_{0}=30 \mu \mathrm{G}$ translates into $\delta=4.1 \times 10^{-2}$ - a factor of ten larger than our fiducial value - with $M_{\Phi, 0} / M_{0}=2.6$.

Figure 11 is a comparison of the evolution of the central density and central magnetic field - this last obtained from evaluating $B$ at $m=10^{-3}$ - between our model and Fiedler \& Mouschovias's. Focusing first on the central density, we see that even though $t_{0}=11.85 \mathrm{Myr}$, the fit is good starting at $t \approx 8 \mathrm{Myr}$ and remains good until $t \approx 16 \mathrm{Myr}\left(t / t_{\mathrm{AD}} \approx 1.8\right)$. After $t=16 \mathrm{Myr}$ the two models diverge, and no fit can be found for any choice of $t_{0}$ and $n_{\mathrm{c}, 0}$. This 
is due to the onset of dynamical collapse in the Fiedler \& Mouschovias calculation, while our model still evolves quasi-statically up to $t \sim 16.5 \mathrm{Myr}$ (refer back to Fig. 6 ). The reason for this discrepancy can be found in the evolution of $B_{c}$ in Figure 11: note how the strength of the central magnetic field increases faster in our model than in Fiedler \& Mouschovias'-by $t \approx 15$ Myr the difference is a factor $\sim 2$, and thereafter we find that $B_{c} \propto n_{c}^{2 / 3}$, i.e. flux-freezing obtains in the core. This larger magnetic field provides enhanced support against gravity and the onset of dynamical collapse is retarded. However it is interesting to note that at $n_{c} \sim 10^{5} \mathrm{~cm}^{-3}$, where our solution starts to differ significantly from that of Fiedler \& Mouschovias, the free-fall time in the core is $\sim 0.1 \mathrm{Myr}$ and the ambipolar diffusion time is $\sim 0.5 \mathrm{Myr}$. Therefore, our solution for $n_{c}$ agrees with that of Fiedler \& Mouschovias up to the point where the evolution of the core becomes dynamical and the core is within a few free-fall times from reaching the origin, notwithstanding the large discrepancy in $B_{c}$. This is so even though the static ion approximation breaks down earlier than in our fiducial cloud-we find that in the core $v_{i} / v_{n} \sim 1$ by $\tau \sim 1.4$ due to the fact that $\delta$ is an order of magnitude larger than the fiducial value used in our numerical examples.

So far we have only proven that our solution compares well with that of Fiedler \& Mouschovias in the central region. Given the discrepancy in the late-time evolution, it is interesting to compare the evolution in other parts of the cloud. Because of the differences between the two calculations, the only meaningful comparison is that between our results and those of Fiedler \& Mouschovias for the midplane density of their cloud; this comparison is presented in Figure 12, which is a plot of density as a function of radius for three different times, $t=10.2 \mathrm{Myr}, t=15.1 \mathrm{Myr}$, and $t=16.0 \mathrm{Myr}$. Two things are noteworthy in this figure. First, even though the central density disagrees by a factor of $\sim 2$, the overall profile is well reproduced . Second, note how the density profile in the envelope is also well reproduced $\left(\rho \propto r^{-2}\right)$, but Fiedler \& Mouschovias's values are consistently larger than ours (again, by $\sim 2$ ); this is a natural consequence of the different geometries. Although Fiedler \& Mouschovias's model is fairly spherical - with an aspect ratio 1:3 in the envelope - our cloud is strictly spherical, so our density in the outer parts has to be lower for the two clouds to have the same total mass. This constraint guided us in choosing $\epsilon=0.1$ for the comparison. Otherwise, for $\epsilon>0.1$, the spatial extent of our core and our envelope density would be larger than those in Fiedler \& Mouschovias's cloud, and the clouds' masses would not match.

These results confirm that the assumptions of negligible thermal stresses and of static ions have minimal consequences for our results for the hydrodynamical variables until the latest stages of gravitational collapse.

\footnotetext{
${ }^{6}$ However, note that for $t=16.0 \mathrm{Myr}$, where the central density is reproduced most accurately, the drop in density at the edge of the core is steeper in the Fiedler \& Mouschovias model than in ours. This is a natural result of neglecting thermal pressure: the density contrast between the center and the edge of a stable, thermally supported cloud is $14: 1$ (Bonnor, 1956), and the mass of the core decreases with increasing density as $\rho^{-1 / 2}$; in our model the mass of the core is fixed, while the density contrast between the center and the edge of the core increases with time from $4: 1$ at $\tau=0$ (eq. 90 ).
} 


\subsection{The Initial Protostellar Mass}

What fraction of the cloud becomes the actual protostar? In our model, the answer is $m_{\mathrm{c}} M_{0}$, which is fixed $a b$ initio once the initial degree of concentration of the cloud $\epsilon$ is fixed. This characteristic mass corresponds to the inner fraction of the cloud that first becomes magnetically supercritical and collapses, detaching itself from the remaining envelope. However, $m_{\mathrm{c}}$ is a free parameter in our model; what determines in nature, then, the protostellar mass? Notwithstanding the shortcomings of our model, here we show that it provides insight to this question.

As we noted before, the stability of a cold cloud is governed by the ratio $M_{\Phi} / M$, where $M_{\Phi}$ is the magnetic critical mass (eq. 480). Those regions of the cloud with $M>M_{\Phi}$ are dynamically unstable and will collapse even if the magnetic field is perfectly coupled to the neutral material. Figure 9 is a plot of $M_{\Phi} / M$ as a function of time and the Lagrangian coordinate $M$ for our fiducial cloud $\left(\epsilon=0.1\right.$ and $\delta=4 \times 10^{-3}$, with $\left.M_{\Phi, 0} / M_{0}=8.4\right)$. Two things are noteworthy in this figure: first, the cloud is initially magnetically subcritical throughout, and as time increases the region $M \sim M_{c}$ first becomes supercritical at $t / t_{\mathrm{AD}} \sim 1.5$. Second, and more important, at any given time the ratio $M_{\Phi} / M$ has a global minimum as a function of $M$, and this minimum occurs at finite $M$. Thus, a region of finite mass first becomes magnetically supercritical, and the collapse proceeds inward from this region. This result is readily understood: for small enough values of $m$, both $B$ and $\rho$ are fairly constant, so that $M_{\Phi} \propto B R^{2} \propto M^{2 / 3}$. On the other hand, in the outer parts of the cloud $\rho \propto r^{-2}$ and $B \sim$ constant, so that $M_{\Phi} \propto R^{2} \propto M^{2}$. Thus altogether $M_{\Phi} / M \propto M^{-1 / 3}+K M$, where $K$ is a constant, and this expression has a minimum at a mass at which the two terms are comparable.

Is this result relevant to clouds with a finite temperature? The answer is yes - as long as magnetic stresses dominate outside the thermally supported core. In a real cloud, there are two characteristic masses, namely a Jeans mass which can be supported by thermal stresses only, $M_{\mathrm{J}}$, and the magnetic critical mass $M_{\Phi}$; and both are time dependent. Therefore, at any time the stability of the cloud is now determined by the ratio $\left(M_{\Phi}+M_{\mathrm{J}}\right) / M(\mathrm{McKee}, 1989)$. If the core is thermally supported, this function will also have a global minimum as a function of $M$. Just as before, this minimum will occur at a mass at which the two terms are comparable -i.e., $M_{\Phi} \sim M_{\mathrm{J}}$ - so that $M$ is of order $M_{\mathrm{J}}$. We conclude that the characteristic protostellar mass is of order the Jean mass at the onset of dynamical evolution (see also Larson 1985, 1995):

$$
\begin{aligned}
M_{\mathrm{J}} & =1.18 \frac{c_{s}^{4}}{\left(G^{3} P_{0}\right)^{1 / 2}} \\
& =3.6\left(\frac{T}{10 \mathrm{~K}}\right)^{2}\left(\frac{P_{0} / k}{10^{4} \mathrm{~K} \mathrm{~cm}^{-3}}\right)^{-1 / 2} \mathrm{M}_{\odot},
\end{aligned}
$$

where $c_{s}$ is the speed of sound and $P_{0}$ is the thermal pressure confining the cloud, which is typically somewhat greater than the thermal pressure in the diffuse ISM (Bertoldi \& McKee 1992). Note that if the core is thermally supported, then the thermal pressure is comparable to the magnetic pressure and the characteristic stellar mass $M_{J} \propto 1 / B$ (cf. Basu \& Mouschovias 1995b). In our 
model, the role of the Jeans mass is taken by the core mass $M_{c}$.

\subsection{The Protostellar Magnetic Flux}

Our model allows us to estimate the initial magnetic flux trapped in the protostellar core, $\Phi_{\text {core }}(\tau=2)$. We have already found the trapped flux analytically for the homogeneous case; here we determine it for the $p=2$ case. We can do this despite the breakdown of the static ion approximation before $\tau=2$ because the breakdown of the approximation leads to $v_{i} \sim v_{n}$, so that flux freezing obtains. As a result, the value of $\Phi_{\text {core }}(\tau=2)$ is essentially the value of $\Phi_{\text {core }}$ at the time the approximation breaks down. This is shown in Figure 13, which is a plot of the evolution of the magnetic flux in the core, $\Phi_{\text {core }}$, in units of the cloud's initial magnetic flux, $\Phi_{0}$, for $\delta=10^{-3}$ and different values of $\epsilon$. Note that the amount of flux trapped in the core at $\tau=2$ is a decreasing function of $\epsilon$, or, equivalently, of $m_{\mathrm{c}}$.

How does $\Phi_{\text {core }}(\tau=2) / \Phi_{0}$ scale with $m_{\mathrm{c}}$ and $\delta$ ? We can answer this question by examining equation (52). To isolate the dependence on $m_{\mathrm{c}}$, let us introduce a new Lagrangian variable $y=m / m_{\mathrm{c}}$. Then, equation (52) reads

$$
\left.\frac{\partial b^{2}}{\partial y}\right|_{\tau}=-\delta m_{\mathrm{c}}^{2} \frac{y}{\hat{r}^{4}} .
$$

According to equation (91), we can write $\hat{r}^{4}=\left(m_{\mathrm{c}} \epsilon\right)^{4 / 3} f(\tau, y)$. Let us assume $m_{c} \ll 1$, so that $\epsilon \simeq 3 m_{c}^{2}$ (eq. 73 with $p=2$ ). We then obtain from equation (96)

$$
b^{2}(\tau, y)=1+\frac{3 \delta}{m_{c}^{2}} \int_{y}^{1 / m_{c}} \frac{y d y}{f(\tau, y)} .
$$

In the core $(y \leq 1)$, and for $\tau>1$, we have $b \gg 1$ (see Figure $5 \mathrm{~b}$ ), and therefore the first term on the r.h.s of equation (97) can be neglected. Furthermore, because the gradient of $b$ is largest just outside the core (again, see Figure 5b), the integral in equation (97) depends weakly on the upper limit of integration, so that

$$
b(\tau>1, y \leq 1) \propto \delta^{1 / 2} m_{c}^{-1} .
$$

As for the magnetic flux in the core, by rewriting equation (29) in Lagrangian coordinates and integrating we obtain, in units of $\Phi_{0}$,

$$
\phi_{\text {core }}=\frac{2}{3} \epsilon m_{\mathrm{c}} \int_{0}^{1} \frac{b d y}{\hat{r} \hat{\rho}} .
$$

Because $\hat{\rho}$ depends on $\tau$ and $y$ only (eq. 90 ) whereas $\hat{r}$ scales as $\left(m_{c} \epsilon\right)^{1 / 3}$, we finally obtain that at late times

$$
\phi_{\text {core }}(\tau>1) \propto m_{\mathrm{c}} \delta^{1 / 2}
$$


The scaling in equation (100) describes our numerical results quite well, and we find the coefficient of proportionality to be 1.16. By analogy with equation (85), we find that the value of $M_{\Phi} / M$ in the core is

$$
\frac{M_{\Phi, \text { core }}}{M_{c}}=\left(\frac{M_{\Phi 0}}{M_{0}}\right) \frac{\phi_{\text {core }}}{m_{c}}=0.61,
$$

independent of $m_{c}$ or $\delta$. Just as in the case of the homogeneous cloud, the flux is reduced until the core is supercritical; the more subcritical the core is to begin with, the greater the flux lost.

\subsection{Post-Collapse Evolution}

A particularly attractive feature of our solution is that we can follow, with the help of equation (90), the evolution of the envelope with $m>m_{c}$ after the collapse of the core at $\tau=2$.

As in the pre-collapse evolution, one should check for self-consistency regarding our quasi-static approximation, the neglect of thermal stresses, and the assumption that $v_{i} \ll v_{n}$. The validity of the quasi-static approximation is assessed with the help of $\alpha$ (eq. 26]), the local ratio of the neutral's acceleration to the gravitational acceleration; similarly, the validity of the $T=0$ approximation is verified by evaluating $\beta^{\prime}$ (eq. [60]). On the other hand, the assumption of negligible motion of the ions requires special consideration.

Verification of the assumption $v_{i} / v_{n} \ll 1$ requires $\Phi(t, r)$ to be known. It is difficult to evaluate $\Phi(t, r)$ after the collapse of the core in the same way as for $\tau<2$ (integrating eq. [29] outwards from $r=0$ ) because, as more and more mass shells accrete onto the protostar, the protostellar magnetic flux increases with time, and its evaluation requires the solution of a partial differential equation. However, one can evaluate the magnetic-flux loss at the edge of the cloud and find $\Phi\left(M=M_{0}\right)$ as a function of time in the following way. Adding and substracting $v_{n} \partial \Phi / \partial r$ to the l.h.s of equation (28), substituting $\partial \Phi / \partial r$ from equation (29), and switching to Lagrangian coordinates we obtain

$$
\frac{D \Phi}{D t}=2 \pi v_{n}\left(1-\frac{v_{i}}{v_{n}}\right) B r \simeq 2 \pi v_{n} B r,
$$

where the last step follows for $v_{i} \ll v_{n}$. Note that at the edge of the cloud we have $B=B_{0}$, so that equation (102) may be integrated to evolve the magnetic flux at $M=M_{0}$. With this value at hand, we can then integrate equation (29) inwards. It turns out that integrating inwards equation (29) with the initial value found from equation (102) starts to breakdown at the point where $v_{i} \sim v_{n}$ (we obtain negative values for $\Phi$; recall that $v_{n}$ is negative definite), but, as long as $\alpha \ll 1$, the assumption $v_{i} \ll v_{n}$ is very good throughout the quasi-static envelope7. Finally, we find that the assumption of negligible thermal stresses is very good throughout the post-collapse

\footnotetext{
${ }^{7}$ The same method may be applied to find $\Phi$ during the pre-collapse stage. We find that as long as $v_{i} \ll v_{n}$ the two methods (integrating outwards from $r=0$ and integrating inwards from $M=M_{0}$ ) agree very well. However, as soon as $v_{i}$ approaches $v_{n}$ in the core, the integration from $M=M_{0}$ breaks down for $m \leq m_{c}$, although it yields $v_{i} \ll v_{n}$ throughout the envelope.
} 
evolution and that our results are only limited by the breakdown of the quasi-static approximation. We arbitrarily deem the evolution to become dynamical where $\alpha \geq 0.1$.

Figure 14 is a plot of the neutral velocity and density in our fiducial cloud as a function of position $r$ for six different times after the collapse of the core; for each curve, the smallest value of $r$ is that where $\alpha=0.1$, while the largest corresponds to the edge of the cloud. For $M_{0}=5 \mathrm{M}_{\odot}$ and $R_{0}=0.45 \mathrm{pc}$ - and recalling that $\epsilon=0.1$-we obtain $n_{c, 0}=2200 \mathrm{~cm}^{-3}$ and $v_{n 0}=4.5 \times 10^{-3} \mathrm{~km} \mathrm{~s}^{-1}$; therefore the typical densities in the envelope are $\gtrsim 750 \mathrm{~cm}^{-3}$ and the maximum velocities are $\sim-0.10 \mathrm{~km} \mathrm{~s}^{-1}$ — a factor $\lesssim 2$ smaller than the speed of sound for $T=10 \mathrm{~K}$.

Two things are noteworthy in Figure 14. First, after the collapse of the core at $t / t_{\mathrm{AD}}=2.0$ the density profile starts to flatten as the velocity profile steepens. This is a natural result of the increased acceleration of the neutrals. Second, the point where the evolution becomes dynamical moves outward with time, and therefore the collapse of the envelope is from inside-out after the collapse of the core (see also Foster \& Chevalier, 1993). For our fiducial solution this collapse front moves at a speed $\sim 0.05 \mathrm{~km} \mathrm{~s}^{-1}\left(R_{0} / 0.5 \mathrm{pc}\right)\left(n_{c, 0} / 10^{3} \mathrm{~cm}^{-3}\right)^{1 / 2}$ - smaller by $\sim 4$ than a typical speed of sound in dark clouds.

\subsection{Mass Accretion Rates}

We can use our results to evaluate the mass flow rates in regions where the flow is quasi-static, and to estimate the accretion rate onto the protostar after it forms. In spherical symmetry the mass accretion rate at any point $r$ in the cloud is given by

$$
\dot{M}=4 \pi r^{2} v_{n} \rho \equiv \dot{M}_{\mathrm{AD}} m \hat{\rho}^{1 / 2},
$$

where we have used the non-dimensional expressions in eqs. (35), (36), (44), and (53). The characteristic accretion rate due to ambipolar diffusion is

$$
\begin{aligned}
\dot{M}_{\mathrm{AD}} & \equiv \frac{M_{0}}{t_{\mathrm{AD}}} \\
& =4.1 \times 10^{-7} \mathrm{M}_{\odot} \mathrm{yr}^{-1}\left(\frac{M_{0}}{5 \mathrm{M}_{\odot}}\right)^{3 / 2}\left(\frac{R_{0}}{0.5 \mathrm{pc}}\right)^{-3 / 2} \epsilon^{-1 / 2}
\end{aligned}
$$

where we have used equations (40) and (46); recall that $\epsilon$ is the initial ratio of mean to central density. Figure 15 is a plot of $\dot{M}$ (in units of the fiducial mass accretion rate $\dot{M}_{\mathrm{AD}}$ ) as a function of radius in our fiducial cloud for both the pre- and post-collapse stages.

Focusing first on the pre-collapse evolution, we note that for our fiducial cloud $(\epsilon=0.1)$ and reasonable values of the cloud's mass $\left(M_{0} \sim\right.$ a few $\left.\mathrm{M}_{\odot}\right)$ and initial radius $\left(R_{0} \lesssim 1 \mathrm{pc}\right)$ the value of $\dot{M}$ for most of the cloud is $\sim$ few $\times 10^{-7} \mathrm{M}_{\odot} \mathrm{yr}^{-1}$. However, at any given time $t / t_{\mathrm{AD}}<2$, $\dot{M}$ has a maximum as a function of $r$; this maximal value increases with time, and its location moves inward with $\dot{M}(r=0) \rightarrow \infty$ as $\tau \rightarrow 2$. This feature is a direct result of our assumed initial condition, which results in a constant core mass as a function of time. 
As for the post-collapse accretion rates, $\dot{M}(r)$ is plotted in Figure 15 up to the point where the quasi-static approximation breaks down $(\alpha \geq 0.1)$. The results in Figure 15 show, again, that to a good degree of approximation the mass accretion rate in the quasi-static envelope $\left(r / R_{0} \gtrsim 0.1\right)$ is constant-with $\dot{M} \sim$ few $\times 10^{-7} \mathrm{M}_{\odot} \mathrm{yr}^{-1}$.

How do these results depend on the free parameters, namely $M_{0}, R_{0}$, and $\epsilon$ ? Using equation (104), equation (103) can be rewritten as

$$
\dot{M}=4.1 \times 10^{-7} \mathrm{M}_{\odot} \mathrm{yr}^{-1}\left(\frac{M_{0}}{5 \mathrm{M}_{\odot}}\right)^{3 / 2}\left(\frac{R_{0}}{0.5 \mathrm{pc}}\right)^{-3 / 2} \epsilon^{-1 / 2} m \hat{\rho}^{1 / 2} .
$$

We find that in the range $0.01 \leq \epsilon \leq 0.2$ the combination $\epsilon^{-1 / 2} m \hat{\rho}^{1 / 2} \sim 0.6-1.6$ (depending on the values of $r / R_{0}$ and $t / t_{\mathrm{AD}}$ where the expression is evaluated); this translates-holding $M_{0}$ and $R_{0}$ constant - into a change $\lesssim 3$ in the mass accretion rates. Therefore, we conclude that for reasonable choices of the initial degree of concentration $(0.01 \leq \epsilon \leq 0.2)$, the cloud's mass $\left(M_{0} \sim\right.$ a few solar masses $)$, and initial cloud radius $\left(R_{0}=0.5-1.0 \mathrm{pc}\right)$ the mass accretion rate in the quasi-static envelope is $\sim$ few $\times 10^{-7} \mathrm{M}_{\odot} \mathrm{yr}^{-1}$.

Finally, we address the question of the mass accretion rate onto the protostar, $\dot{M}_{*}$. By continuity, one would expect $\dot{M}_{*}$ to be comparable to the mass accretion rate in the quasi-static envelope. We can answer this question more quantitatively with the help of our expression for $r(t, M)$ (see eq. 91]). Setting $\hat{r}=0$ in equation (91), we can solve for the instantaneous value of $m$ at the origin, i.e., the protostellar mass as a function of time; and by computing its time derivative, obtain $\dot{M}_{*}(t)$. Since we cannot treat the dynamical stage of evolution, this procedure will become reasonably accurate only after about one free fall time after the collapse. However, although we cannot treat the rate of accretion accurately during this stage, we can obtain a good estimate for the integrated accretion-i.e., the total mass of the protostar that forms during the dynamical phase. Figure 16 is a plot of $M_{*}$ and $\dot{M}_{*}$ (in units of, respectively, $M_{0}$ and $\dot{M}_{\mathrm{AD}}$ ) as a function of time after the formation of the protostar for various values of $\epsilon$.

In our model, the formation of the initial protostar corresponds to an infinite accretion rate. For $\tau-2 \ll 1$, we find that $M_{*} / M_{0}-1 \propto(\tau-2)^{1 / 2}$, and therefore $\dot{M}$-and the accretion luminosity - diverge as $(\tau-2)^{-1 / 2}$ (though this is an integrable singularity, and the total energy radiated by the shock vanishes as $\left.(\tau-2)^{1 / 2}\right)$. However, this is an artifact due to neglecting thermal stresses. Recall that in our model the protostellar mass is a discontinous function of time near $\tau=2$, with $M_{*}=0$ as $\tau \longrightarrow 2^{-}$and $M_{*}=m_{c} M_{0}$ as $\tau \longrightarrow 2^{+}$; the infinite accretion rate is a result of this discontinous behavior. When thermal pressure is included, it provides a retarding force whose effect makes the protostellar mass a continous function of time at $\tau=2$.

\footnotetext{
${ }^{8}$ For $\tau \geq 2$ - and as a result of ignoring thermal pressure $-\hat{r}=0$ is a singular point of equation (51). However, we can still find $M_{*}(\tau)$ from equation $(91$ by setting $\hat{r}=0$ because a real protostar will have a finite radius. For a typical protostellar radius $R_{*} \sim 5 \mathrm{R}_{\odot}$ and an initial cloud radius $R_{0} \sim 0.5 \mathrm{pc}$, the lhs. of equation (91) evaluated at the protostellar boundary is of order $10^{-20}$ —or zero, for all practical purposes.
} 
The calculations of Foster \& Chevalier (1993) illuminate this point. They followed the collapse of a non-singular cloud when only thermal pressure opposes gravity; therefore, their results are directly applicable to our phase of dynamical infall near $\tau=2$. They find that $\dot{M}$ is finite at the instant the protostar is formed, and it rises very sharply immediately thereafter. After this initial rise, which lasts for about a tenth of a free-fall time, the accretion rate declines in very much the same way as in our model.

For our fiducial choice of parameters, a tenth of a free-fall time at the density of dynamicalinfall onset corresponds to $(\tau-2) \approx 2 \times 10^{-3}$, or $t \lesssim 10^{4}$ yr after the formation of the initial protostar. Assuming a radius for the hydrostatic protostellar core $R_{*}=5 R_{\odot}$, we find an accretion luminosity

$$
L_{\mathrm{acc}}=230 L_{\odot} m_{c}^{2} \epsilon^{-1 / 2}\left(\frac{\tau-2}{2 \times 10^{-3}}\right)^{-1 / 2}\left(\frac{M_{0}}{5 M_{\odot}}\right)^{5 / 2}\left(\frac{R_{0}}{0.5 \mathrm{pc}}\right)^{-3 / 2}\left(\frac{R_{*}}{5 R_{\odot}}\right)^{-1} .
$$

For our fiducial model $\left(\epsilon=0.1\right.$ and $m_{c}=0.26$, which corresponds, for $M_{0}=5 M_{\odot}$, to an initial protostar of $1.3 M_{\odot}$ ), we find that the accretion luminosity $\approx 10^{4} \mathrm{yr}$ after the formation of the initial protostar is $49 L_{\odot}$. For an initial protostellar mass $M_{*}=0.5 \mathrm{M}_{\odot}$ (obtained with $\epsilon=0.025$, which gives $m_{c}=0.1$ ), we find, for the same time after the initial collapse, an accretion luminosity $\approx 15 L_{\odot}$. This last value is only a factor $\sim 2$ higher than those of typical Class 0 sources (Gregersen et al., 1997).

As shown in Figure 16, the mass accretion rate remains very high for about a free fall time $\left(\sim 0.2 t_{\mathrm{AD}}\right)$ after the collapse, and, for the range in $\epsilon$ explored, the protostellar mass increases by a factor $\sim 2$ during this phase. Thereafter, $\dot{M}_{*}$ is comparable to the mass accretion rates in the quasi-static envelope, as expected. This strong dependence of $\dot{M}_{*}$ shortly after the formation of the protostar has also been found by Foster \& Chevalier (1993) and Hunter (1977) for the case of non-magnetic collapse - a result that is at strong variance with the predictions from the singular isothermal sphere model (Shu 1977). The later stages of accretion are more in accord with the constant accretion rate expected in the singular isothermal sphere model; particularly - when one takes into account that $\dot{M}_{\mathrm{AD}} \propto \epsilon^{-1 / 2}$ (eq. 104]) $-\dot{M}_{*}$ is essentially independent of $\epsilon$ during the post-collapse, quasi-static phase. This similarity with the singular isothermal sphere model merits further attention, and we address it in the next section

\subsubsection{The Post-Collapse Mass Accretion Rate at Late Times and the Singular Isothermal Sphere Model}

The standard paradigm for the gravitational collapse of an interstellar cloud is the inside-out collapse of a singular isothermal sphere (Shu 1977). The cloud is assumed to be initially in an unstable equilibrium in which gravity is balanced by the pressure of an isothermal gas so that $\rho=c^{2} / 2 \pi G r^{2}$, where $c$ is the isothermal sound speed. Collapse begins at the origin, where the dynamical time is the smallest, and an expansion wave moves outward at $c$. At a time $t$ 
after collapse begins, a mass $2 c^{3} t / G$ has been swept up by the expansion wave; of this, a mass $0.975 c^{3} t / G$ is in a condensed core at the origin.

In light of our results for the post-collapse, quasi-static accretion rate - namely, that at late times after the collapse of the inner core of a non-singular stratified cloud the mass accretion rate onto the protostar approaches a constant value - we wish to compare our predicted accretion rates at late times with that predicted by the singular isothermal sphere model. To do this, we note that at $\tau=2$ the density distribution of the envelope is given by $\rho \propto r^{-1 / 2}$ (see Figure 14), the same density distribution as the singular isothermal cloud. At late times $(\tau \gg 1)$, the mass shells that still are in quasistatic equilibrium satisfy $m \gg m_{\mathrm{c}}$; in this limit, equation (72) for $m(r)$ reduces to $m=\hat{r}$, and the initial density distribution (eq. [26]) after core collapse becomes $\hat{\rho}(\tau=0)=\left(m_{\mathrm{c}} / m\right)^{2}$. Rather than measuring time in units of the central ambipolar diffusion time $t_{\mathrm{AD}}$, we use now the ambipolar diffusion time at the surface, $t_{\mathrm{AD}}^{\prime}=t_{\mathrm{AD}}\left[\rho_{c 0} / \rho(0,1)\right]^{1 / 2}=t_{\mathrm{AD}} / m_{\mathrm{c}}$. The density distribution becomes

$$
\frac{\rho\left(\tau^{\prime}, m\right)}{\rho(0,1)}=\frac{4}{\left[m-\tau^{\prime}+\left(m^{2}-2 m \tau^{\prime}\right)^{1 / 2}\right]^{2}} \quad\left(m \gg m_{\mathrm{c}}\right)
$$

where $\tau^{\prime} \equiv t / t_{\mathrm{AD}}^{\prime}=m_{\mathrm{c}} \tau$ and $\tau^{\prime}=0$ corresponds to the formation of a singularity at the origin. The radius of the mass shell $m$ is

$$
\hat{r}^{3}=\frac{1}{2} m^{3}-\frac{3}{2} m^{2} \tau^{\prime}+\frac{3}{4} m \tau^{\prime 2}+\frac{1}{2}\left(m^{2}-2 m \tau^{\prime}\right)^{3 / 2} \quad\left(m \gg m_{\mathrm{c}}\right) .
$$

This equation indicates that the shell reaches the origin at a time $\tau_{\mathrm{cr}}^{\prime}=\frac{4}{9} m$. By this time, our approximations for the dynamics have broken down, but this nonetheless defines a characteristic time scale for the evolution. At this time, equation (107) shows that the density has increased by a factor of about 5 .

If we approximate the acceleration of the gas by $v_{n}^{2} / r$, the parameter $\alpha$ (eq. 26]) becomes

$$
\alpha \simeq \frac{4 \pi G}{k_{n i}^{2}}\left[\frac{r(0, m)}{r\left(\tau^{\prime}, m\right)}\right]^{3}\left[\frac{\rho(0, m)}{\rho\left(\tau^{\prime}, m\right)}\right] .
$$

As discussed in $\S 5.2 .1, \alpha$ is initially very small; it will begin to approach unity (so that our quasi-static approximation breaks down) only when $\hat{r}^{3}$ becomes significantly smaller than its initial value. Equation (108) shows that this will occur only when $\tau^{\prime}$ is quite close to $\tau_{\text {cr }}^{\prime}$. It is therefore self-consistent to use to use $\tau_{\text {cr }}^{\prime}$ as an estimate for the evolution time for the shell.

The time scale for evolution $\tau_{\text {cr }}^{\prime}$ enables us to define the characteristic velocity with which ambipolar diffusion advances into the cloud,

$$
v_{\mathrm{AD}} \equiv \frac{r(0, m)}{\tau_{\mathrm{cr}}^{\prime} t_{\mathrm{AD}}^{\prime}}=\frac{9}{4} \frac{R_{0}}{t_{\mathrm{AD}}^{\prime}},
$$

which is constant. How does this compare with the expansion wave associated with the collapse of an unmagnetized, singular isothermal sphere, which moves outward at the sound speed $c$ ? 
Defining an effective sound speed through the relation governing such a sphere, $c^{2}=2 \pi r^{2} G \rho$, and using equation (40), we find

$$
v_{\mathrm{AD}}=\frac{3.2 c}{\nu_{\mathrm{ff}}} .
$$

Thus, for typical conditions in a shielded core in which the ionization is dominated by cosmic rays, so that $\nu_{\mathrm{ff}} \sim 10$, the ambipolar diffusion advances into the envelope at about $1 / 3$ the speed of the expansion wave. The result is not changed significantly if we instead compare our result with that for the collapse of a thin disk with a frozen-in magnetic field: Li \& Shu (1997) have shown that in this case the expansion wave advances at $c \sqrt{ } 2$, about the same velocity as in the unmagnetized case.

The accretion rate is given by equation (101). Evaluating this rate at the time $\tau_{\mathrm{cr}}^{\prime}$, we find

$$
\dot{M}=\frac{9}{4}\left(\frac{M_{0}}{t_{\mathrm{AD}}^{\prime}}\right)=5.4 \times 10^{-7}\left(\frac{M_{0}}{5 M_{\odot}}\right)^{3 / 2}\left(\frac{R_{0}}{0.5 \mathrm{pc}}\right)^{-3 / 2} \quad M_{\odot} \mathrm{yr}^{-1},
$$

in good agreement with the estimate for the non-singular case in $§ 5.7$. If we express the accretion rate in terms of the sound speed required to support the sphere, as we did in equation (111), we find

$$
\dot{M}=\frac{6.38}{\nu_{\mathrm{ff}}}\left(\frac{c^{3}}{G}\right) .
$$

Recall that for the non-magnetized singular isothermal sphere, the accretion rate is $\dot{M}=0.975 c^{3} / G$ (Shu 1977). Our results indicate that when ambipolar diffusion is included, the accretion rate is reduced only slightly: for $\nu_{\mathrm{ff}} \sim 10$, we obtain an accretion rate that is about $2 / 3$ that for the equivalent non-magnetized case.

This result is quite remarkable: it indicates that the presence of a strong magnetic field does not significantly reduce the accretion rate onto a protostar at late times in a cloud in which the ionization is due to cosmic rays (photoionization by FUV or X-ray photons increases $\nu_{\mathrm{ff}}$ and reduces the accretion rate proportionately). To see why this is so, let us first estimate the accretion rate for the singular isothermal sphere. Since about half the swept up mass has collapsed to the origin at any time, the accretion rate is $\dot{M}_{\text {SIS }} \sim M / 2 t_{\mathrm{ff}}$, where $t_{\mathrm{ff}}$ is the free fall time based on the mean density $\bar{\rho}=3 \rho_{0}$. In terms of $t_{\mathrm{ff}}{ }^{\prime}$, the free fall time at $\rho_{0}$, we then have

$$
\dot{M}_{\mathrm{SIS}} \sim \frac{\sqrt{ } 3}{2}\left(\frac{M}{t_{\mathrm{ff}^{\prime}}}\right)=\frac{4}{\pi}\left(\frac{c^{3}}{G}\right) .
$$

This is somewhat higher than the exact answer, presumably because we neglected the retarding effects of the gas pressure.

Next, consider the magnetized case. For a uniform cloud, the average accretion rate is $\dot{M} \sim M / 2 t_{\mathrm{AD}}$ based on the results in $\S 4$. Since the mass of a singular sphere is 3 times that of a uniform sphere with the same density at the surface, the ambipolar diffusion velocity, and hence 
the collapse rate, will be three times greater: $\dot{M}_{\mathrm{SIS}, \mathrm{AD}} \sim \frac{3}{2}\left(M / t_{\mathrm{AD}}{ }^{\prime}\right)$, which is within a factor 1.5 of the exact answer in equation (112). With these estimates, we find

$$
\frac{\dot{M}_{\mathrm{SIS}, \mathrm{AD}}}{\dot{M}_{\mathrm{SIS}}} \sim \frac{3 M / 2 t_{\mathrm{AD}^{\prime}}}{\sqrt{ } 3 M / 2 t_{\mathrm{ff}^{\prime}}}=\sqrt{ } 3\left(\frac{t_{\mathrm{ff}}{ }^{\prime}}{t_{\mathrm{AD}^{\prime}}}\right)=\frac{\sqrt{ } 3}{\left(\frac{8}{3 \pi^{2}}\right)^{1 / 2} \nu_{\mathrm{ff}}} .
$$

With allowance for the fact that our estimate for this ratio is off by a factor 2 (the estimate for the magnetized case was somewhat too low, and that for the unmagnetized case was too high), this agrees with the result in equation (113). Several factors have raised the magnetized accretion rate close to the unmagnetized rate: in the magnetized case, the entire cloud is contracting at $t=2 t_{\mathrm{AD}}$, whereas in the unmagnetized case the collapse begins only after the expansion wave reaches the point in question; thermal pressure retards the unmagnetized case somewhat (we have assumed that magnetic pressure dominates thermal pressure in the magnetized case); and finally, in going from an estimate based on a uniform sphere to a singular sphere, the rate for the magnetized case increases by $\bar{\rho} / \rho_{0}=3$, whereas that for the unmagnetized case increases only by the square root of this ratio.

We conclude that, contrary to one's expectation (Mouschovias 1991), the accretion rate onto a protostar at late times after its formation is not significantly inhibited by the presence of a strong magnetic field, so long as the ionization is due to Galactic cosmic rays. Note that this result does not validate the singular isothermal model in toto. It only means that at late times, after the formation of the protostar, the mass accretion rate onto the central object approaches the limiting case of a singular isothermal cloud. We must stress, again, that our results for core collapse and immediately thereafter are at strong variance with the predictions of the singular isothermal sphere model.

In our idealized model, the accretion will continue until the mass of the envelope is exhausted. In reality, several other factors could intervene: the outer envelope is likely to be photoionized (McKee 1989), with a much longer ambipolar diffusion time, and the outflow from the protostar could disperse the envelope (Shu et al 1987).

\section{Conclusions}

In this work we have investigated the evolution of a cold, magnetized molecular cloud by means of an idealized, semi-analytic model. We neglect thermal pressure, assume quasi-static evolution, and impose spherical symmetry. Our principal conclusions are the following:

1. In the absence of thermal stresses, an initially homogeneous collapses homologously, without developing any structure. The collapse reaches a singularity at a time $2 t_{\mathrm{AD}}$, where $t_{\mathrm{AD}}$ is the initial ambipolar diffusion time. 
2. The central core of a centrally condensed cloud also collapses after a time $2 t_{\mathrm{AD}}$ (where $t_{\mathrm{AD}}$ is measured at the center of the cloud), leaving behind a quasi-static envelope.

3. In a centrally condensed cloud the collapse of the core proceeds from outside-in, while the quasi-static envelope collapses from inside-out after the collapse of the central region.

4. The pre-collapse evolution of the core is similar to the evolution of a homogeneous cloud. In particular, the quasi-static evolution of the central density is a universal function of time, $\rho_{c} \propto\left(2-t / t_{\mathrm{AD}}\right)^{-2}$, independent of any parameters that enter the calculation.

5. The core evolves quasi-statically up to the point that it becomes magnetically supercritical. Thereafter, the ions are no longer approximately static, and our approximations break down. As a result, we cannot treat the subsequent dynamical collapse. Nonetheless, even after the formation of the central protostar, we are able to follow the quasi-static evolution of the envelope so long as it remains magnetically subcritical.

6. Most of the initial magnetic flux in the core is lost via ambipolar diffusion; after collapse, we estimate that the remaining trapped flux is about half the critical value.

7. The mass accretion rate in the quasi-static envelope before and after core collapse is remarkably constant and of order

$$
\dot{M} \sim \text { few } \times 10^{-7} \mathrm{M}_{\odot} \mathrm{yr}^{-1}\left(\frac{M_{0}}{5 \mathrm{M}_{\odot}}\right)^{3 / 2}\left(\frac{R_{0}}{1.0 \mathrm{pc}}\right)^{-3 / 2}\left(\frac{\epsilon}{0.1}\right)^{-1 / 2}
$$

where $M_{0}$ and $R_{0}$ are, respectively, the cloud's mass and inital radius and $\epsilon$ is the inital ratio of mean to central density, with $0.01 \lesssim \epsilon \lesssim 0.2$.

8. Much of the mass of the star is built up during the dynamical phase of accretion, which lasts about a free-fall time. Although we cannot treat this dynamical phase, our model does enable us to determine the total mass accreted and to follow the subsequent quasi-static accretion. Just as in the non-magnetic case (Foster \& Chevalier 1993), the accretion rate during this stage is much larger than that of a singular isothermal sphere.

9. The late-time mass accretion rate onto the protostar is quasi-static. Notwithstanding the inhibiting effects of the magnetic field, the accretion rate becomes comparable to that of a singular isothermal sphere.

10. The excellent agreement between our results for the pre-collapse evolution and the detailed numerical work of Fiedler \& Mouschovias (1992) indicates that our simple approach captures the basic physics relevant to the evolution of a cloud undergoing ambipolar diffusion. Our results are especially relevant to the late stages of cloud evolution, when thermal stresses are relatively small. Since thermal pressure does play an important role at earlier times, particularly in creating the initial density distribution, our intention is to extend the present calculation to one that incorporates thermal pressure in a subsequent paper. 
The research of PNS was supported by a NASA grant to the Center for Star Formation Studies and by NSF grant AST9314847 to the Laboratory for Millimeter-Wave Astronomy at the University of Maryland; the research of CFM is supported by the National Science Fundation (AST95-30480) and by a NASA grant to the Center for Star Formation Studies; and SWS is supported by the NASA Astrophysics Theory Program, grant NAGW-3107. 


\section{REFERENCES}

Benson, P. J., \& Myers, P. C. 1989, ApJS, 71, 89

Basu, S., \& Mouschovias T. Ch. 1994, ApJ, 432, 720

Basu, S., \& Mouschovias T. Ch. 1995a, ApJ, 452, 386

Basu, S., \& Mouschovias T. Ch. 1995b, ApJ, 453, 271

Bertoldi, F., \& McKee, C. F. 1992, ApJ, 395, 140

Bodenheimer, P., \& Sweigart, A. 1968, ApJ, 152, 515

Bodenheimer, P., \& Black, D. C. 1978, in Protostars \& Planets, ed. T. Gehrels (Tucson:University of Arizona Press), pg. 288

Bonnor, W. B. 1956, MNRAS, 116, 351

Boss, A. P., \& Myhill, E. A. 1993, ApJS, 83, 311

Caselli P. \& Myers P. C. 1995, ApJ, 446, 665

Cernicharo, J. 1991, in The Physics of Star Formation and early Stellar Evolution, eds. C. J. Lada \& N. D. Kylafis (Dordrecht:Kluwer), pg. 287

Chiueh, T., \& Chou, J. 1994, ApJ, 431, 380

Elmegreen, B. G. 1979, ApJ, 232, 729

Ciolek, G. E., \& Mouschovias T. Ch. 1993, ApJ, 418, 774

Ciolek, G. E., \& Mouschovias T. Ch. 1994a, ApJ, 421, 561

Ciolek, G. E., \& Mouschovias T. Ch. 1994b, ApJ, 425, 142

Ciolek, G. E., \& Mouschovias T. Ch. 1995, ApJ, 454, 194

Field, G. B. 1965, ApJ, 142, 531

Fiedler, R., \& Mouschovias, T. C. 1992, ApJ, 415, 680

Foster, P. N., \& Chevalier, R. A. 1993, ApJ, 416, 303

Galli, D., \& Shu, F. H. 1993a, ApJ, 417, 220

Galli, D., \& Shu, F. H. 1993b, ApJ, 417, 243

Goldreich, P., \& Weber, S. V. 1980, ApJ, 238, 991

Gregersen E. M. , Evans II N. J., Zhou S., \& Choi M. 1997, preprint

Heiles, C. 1987, in Physical Processes in Interstellar Clouds, eds. G. E. Morfill \& M. Scholer (Dordrecht:Reidel), pg. 429

Holliman, J., \& McKee, C. M. 1996, in preparation

Hunter, C. 1977, ApJ, 218, 834

Larson, R. B. 1969, MNRAS, 145, 271 
Larson, R. B. 1985, MNRAS, 214, 379

Larson, R. B. 1995, MNRAS, 272, 213

Li, Z.-Y., \& Shu, F. H. 1996, ApJ, 000, 000

Lizano, S., \& Shu, F. H. 1989, ApJ, 342, 834

McDaniel, E. W., \& Mason, E. A. 1973, The Mobility and Diffusion of Ions in Gases (New York: Wiley)

McKee, C. F. 1989, ApJ, 345, 782

McKee, C. F., Zweibel, E. G., Goodman, A. A., \& Heiles, C. 1993, in Protostars and Planets III, eds. E. H. Levy \& J. I. Lunine (Tucson: University of Arizona Press), pg. 327

Mestel, L., \& Spitzer, L., Jr., 1956, MNRAS, 116, 505

Mouschovias, T. Ch. 1976a, ApJ, 206, 753

Mouschovias, T. Ch. 1976b, ApJ, 207, 141

Mouschovias, T. Ch. 1978, in Protostars \& Planets, ed. T. Gehrels (Tucson:University of Arizona Press), pg. 209

Mouschovias, T. Ch. 1979, ApJ, 228, 475

Mouschovias, T. Ch. 1991, ApJ, 373, 169

Mouschovias, T. Ch. 1996, in Solar and Astrophysical Magnetohydrodynamic flows, ed. K. Tsinganos (Dordrecht:Kluwer), in press

Mouschovias, T. Ch., \& Spitzer, L. Jr. 1976, ApJ, 210, 326

Mouschovias, T. Ch., Paleologou, E. V., \& Fiedler, R. A. 1985, ApJ, 291, 772

Mouschovias, T. Ch., \& Morton, S. A. 1991, ApJ, 371, 296

Myers, P. C. 1985, in Protostars and Planets II, eds. D. C. Black \& M. S. Matthews (Tucson:University of Arizona Press), pg. 81

Myers, P. C., Fuller, G. A., Goodman, A. A., \& Benson, P. J. 1991, ApJ, 376, 561

Myers, P. C., \& Kershonsky, V. K. 1995, ApJ, 442, 186

Myhill, E. A., \& Boss, A. P. 1993, ApJS, 89, 345

Nakano, T. 1979, PASJ, 31, 697

Nakano, T. 1982, PASJ, 34, 337

Nakano, T. 1983, PASJ, 35, 209

Paleologou, E. V., \& Mouschovias, T. Ch. 1983, ApJ, 275, 838

Penston, M. V. 1969, MNRAS, 144, 425

Ryden, B. S. 1996, ApJ, 000, 000

Scott, E. H. 1984, ApJ, 278, 396 
Scott, E. H., \& Black, D. C. 1980, ApJ, 239, 166

Shu, F. H. 1977, ApJ, 214, 488

Shu, F. H. 1983, ApJ, 273, 202

Spitzer, L. Jr. 1978, Physical Processes in the Interstellar Medium (New York: Wiley), pg. 286

Strittmater, P. A. 1966, MNRAS, 132, 359

Tomisaka, K., Ikeuchi, S., \& Nakamura, T. 1988a, ApJ, 326, 208

Tomisaka, K., Ikeuchi, S., \& Nakamura, T. 1988b, ApJ, 335, 239

Tomisaka, K., Ikeuchi, S., \& Nakamura, T. 1989, ApJ, 341, 220

Tomisaka, K., Ikeuchi, S., \& Nakamura, T. 1990, ApJ, 362, 202

Tomisaka, K. 1991, ApJ, 376, 190

Zhou, S., Evans, N. J. III, Wang, Y., Peng, R., \& others 1994, ApJ, 433131

Zuckerman, B., \& Palmer, P. 1974, ARA\&A, 12, 279 
Fig. 1. - Evolution of the Lagrangian radius $r$ (in units of the initial cloud radius $R_{0}$ ) as a function of time for a homogeneous cloud with $\delta=4 \times 10^{-3}$. The five curves show the evolution of $r$ for five different values of the dimensionless Lagrangian coordinate $m: m=10^{-2}, 3.16 \times 10^{-2}, 10^{-1}$, $3.16 \times 10^{-1}$, and 1 . All the masses are in units of the cloud's mass $M_{0}$, and time is in units of the characteristic ambipolar diffusion time, $t_{\mathrm{AD}}$.

Fig. 2. - Evoution of the magnetic critical ratio $M_{\Phi} / M$ for a homogeneous cloud with $\delta=4 \times 10^{-3}$. (a): $M_{\Phi} / M$ as a function of time (in units of the ambipolar diffusion time $t_{\mathrm{AD}}$ ) for three different values of the dimensionless Lagrangian coordinate $m: m=10^{-2}, m=10^{-1}$, and $m=1.0$. (b): $M_{\Phi} / M$ as a function of the dimensionless coordinate $M / M_{0}$ at three different times (in units of the characteristic ambipolar diffusion time): $\tau=0.0,1.62$, and 1.86 .

Fig. 3.- Ratio of the ion's velocity $v_{i}$ to the neutral's velocity $v_{n}$ for a homogeneous cloud with $\delta=4 \times 10^{-3}$. Shown is $v_{i} / v_{n}$ as a function of the dimensionless Lagrangian coordinate $M / M_{0}$ at four different times (in units of the characteristic ambipolar diffusion time): $\tau=0.0,1.00,1.62$, and 1.86 .

Fig. 4. - The mass of the initial central core, $M_{\mathrm{c}}$ (in units of $M_{0}$ ), as a function of the initial degree of concentration, $\epsilon=\langle\rho\rangle / \rho_{\mathrm{c}, 0}$ for a stratified cloud with $p=2$. Only values $m_{c}=M_{\mathrm{c}} / M_{0} \leq 1$ are physically relevant. Clouds with $\epsilon \geq 3 / 7$ have $m_{\mathrm{c}} \geq 1$, and therefore collapse as a homogeneous cloud.

Fig. 5. - Lagrangian radius $r$, neutral velocity $v_{n}$, density $\rho$, and magnetic field $B$ for our fiducial stratified cloud with an initial degree of concentration $\epsilon=0.1$ and magnetic parameter $\delta=4 \times 10^{-3}$ at five different times (in units of the characteristic ambipolar diffusion time $t_{\mathrm{AD}}$ ): $\tau=0.0,0.88$, 1.37, 1.64, and 1.80 (between consecutive values of $\tau$ the central density increases by a factor of $\left.(10)^{1 / 2}\right)$. (a): Lagrangian radius $r$ (in units of the cloud's initial radius $R_{0}-t o p$ panel) and neutral velocity $v_{n}$ (in units of the fiducial velocity $v_{n, 0}$-bottom panel) as a function of the dimensionless Lagrangian coordinate $M / M_{0}$. Time increases from top to bottom for the $r$-curves and from bottom to top for $v_{n}$. The dashed line indicates the location of the central core. (b): Density in units of the initial central density $\rho_{\mathrm{c}, 0}\left(t o p\right.$ panel) and magnetic field (plotted as $B / B_{0}-1$, where $B_{0}$ is the strength of the magnetic field at the edge of the cloud; bottom panel) as functions of the dimensionless Lagrangian coordinate $M / M_{0}$. Time increases from bottom to top. The dashed line indicates the location of the central core.

Fig. 6. - The acceleration of the neutrals in units of the local gravitational acceleration. Shown is $\alpha$, for our fiducial cloud, as a function of the dimensionless Lagrangian coordinate $M / M_{0}$ and dimensionless reversed time (measured from the formation of a singularity at the origin) $2-t / t_{\mathrm{AD}}$.

Fig. 7.- Time evolution of the magnetic flux $\Phi$ in our fiducial cloud (in units of the fiducial magnetic flux $\pi B_{0} R_{0}^{2}$ ) as a function of the dimensionless Lagrangian coordinate $M / M_{0}$ (left panel) and the Eulerian radial coordinate $R$ (in units of the cloud's initial radius $R_{0}$; right panel). Each curve corresponds to a different dimensionless time $\tau(\tau=0.0,0.88,1.37,1.64$, and 1.80), which 
increases from top to bottom in the left panel and from bottom to top in the right panel. The dashed line and the arrows indicate the location of the central core; the arrows are labeled by the value of $t / t_{\mathrm{AD}}$.

Fig. 8.- Ratio of the ion to neutral velocity, $v_{i} / v_{n}$ for our fiducial cloud $\left(\epsilon=0.1\right.$ and $\left.\delta=4 \times 10^{-3}\right)$, as a function of the dimensionless Lagrangian coordinate $M / M_{0}$ for five different times (in units of the characteristic ambipolar diffusion time $t_{\mathrm{AD}}$; from bottom to top): $\tau=0.0,0.88,1.37,1.64$, and 1.80. The dashed line indicates the location of the central core

Fig. 9. - Evolution of the critical ratio $M_{\Phi} / M$ for our fiducial cloud $\left(\epsilon=0.1\right.$ and $\left.\delta=4 \times 10^{-3}\right)$. $M_{\Phi} / M$ is plotted as a function of the dimensionless Lagrangian coordinate $M / M_{0}$ for five different times (in units of the characteristic ambipolar diffusion time $t_{\mathrm{AD}}$; from top to bottom): $\tau=0.0$, $0.88,1.37,1.64$, and 1.80 .

Fig. 10.- Ratio of thermal to magnetic stresses, $\beta^{\prime}$, as a function of the dimensionless Lagrangian coordinate $M / M_{0}$ and dimensionless reversed time (measured from the formation of a singularity at the origin) $2-t / t_{\mathrm{AD}}$. Shown are the results for our fiducial cloud, $\epsilon=0.1$ and $\delta=4 \times 10^{-3}$, with initial central number density $n_{c, 0}=2200 \mathrm{~cm}^{-3}$, magnetic field strength $B_{0}=30 \mu \mathrm{G}$ at the edge of the cloud, and temperature $T=10 \mathrm{~K}$.

Fig. 11. - Comparison with the numerical results of Fiedler \& Mouschovias (1992) for the time evolution of the central number density $n_{c}$ (top panel) and central magnetic field $B_{c}$ (bottom panel) in a cloud with mass $M_{0}=45.5 \mathrm{M}_{\odot}$, initial radius $R_{0}=0.75 \mathrm{pc}$, and external magnetic field $B_{0}=30 \mu \mathrm{G}$. Shown are the results of Fiedler \& Mouschovias for their Model 1 (filled circles and dashed line) after the initial relaxation and up to the onset of dynamical contraction and those from our semi-analytic model (solid line).

Fig. 12. - Comparison with the numerical results of Fiedler \& Mouschovias (1992) for the evolution of the density profile. Shown is the number density as a function of dimensionless radius $r / R_{0}$ for three different times during the quasi-static phase. The filled circles are the numerical results for the midplane of Fiedler \& Mouschovias's Model 1 (light grey: $t=10.2 \mathrm{Myr}$, dark grey: $t=15.1 \mathrm{Myr}$, and black: $t=16.0 \mathrm{Myr}$ ); the solid lines are the results from our semi-analytic model. For easier visualization, the curves have been shifted horizontally by a constant $C: C=\log 0.5$ for $t=10.0 \mathrm{Myr} ; C=\log 0.75$ for $t=15.1 \mathrm{Myr}$; and $C=0.0$ for $t=16.0 \mathrm{Myr}$.

Fig. 13.- Core's magnetic flux $\Phi_{\text {core }}$ (in units of the cloud's initial magnetic flux $\Phi_{0}$ ) as a function of dimensionless reversed time (measured from the formation of a singularity at the origin) $2-t / t_{\mathrm{AD}}$. Shown is $\Phi_{\text {core }} / \Phi_{0}$ for $\delta=10^{-3}$ and four different values of $\epsilon: \epsilon=10^{-4}, 10^{-3}, 10^{-2}$, and $10^{-1}$.

Fig. 14.- Post-collapse evolution of the density profile (top panel) and neutral velocity profile (bottom panel) for our fiducial cloud $(\epsilon=0.1)$. Shown are the density in units of the initial central density and the neutral velocity in units of the fiducial velocity $v_{n, 0}$ as a function of radius in the cloud (in units of the initial cloud radius $R_{0}$ ) for six different times: $\tau=2.0,2.1,2.2,2.3,2.4$, and 
2.5. Time increases from the topmost curve down. The inner boundary of each curve corresponds to the point where the acceleration of the neutrals is $10 \%$ of the local gravitational acceleration.

Fig. 15. - Mass accretion rates as a function of radius for our fiducial cloud $(\epsilon=0.1)$. Shown is $\dot{M}$ (in units of the fiducial mass accretion rate $\dot{M}_{\mathrm{AD}}$ ) as a function of radius in the cloud (in units of $R_{0}$ ) before the collapse of the inner core (dotted lines) and afterwards (solid line and shaded region). For the pre-collapse accretion rates each curve corresponds to a different time (in units of the characteristic ambipolar diffusion time $t_{\mathrm{AD}}$; from bottom to top): $\tau=0.0,0.88,1.37,1.64$, and 1.80. The solid line is the accretion rate at the time when the core collapses $(\tau=2.0)$ and the shaded region corresponds to the post-collapse mass accretion rates for $\tau \leq 2.5$; the individual curves in this regime are too close to each other to show clearly on the scale of this plot. The post-collapse curves $(\tau \geq 2.0)$ are truncated to the left at the point where the acceleration of the neutrals is $10 \%$ of the local gravitational acceleration.

Fig. 16. - Protostellar mass $M_{*}$ and mass accretion rate onto the protostar, $\dot{M}_{*}$, as functions of dimensionless time (measured from the formation of a singularity at the origin) $t / t_{\mathrm{AD}}-2$. Shown are $M_{*}$ (in units of the cloud's mass $M_{0}$; top panel) and $\dot{M}_{*}$ (in units of the fiducial mass accretion rate $\dot{M}_{\mathrm{AD}}$; bottom panel) for three different values of $\epsilon: \epsilon=0.05,0.1$, and 0.2 . 


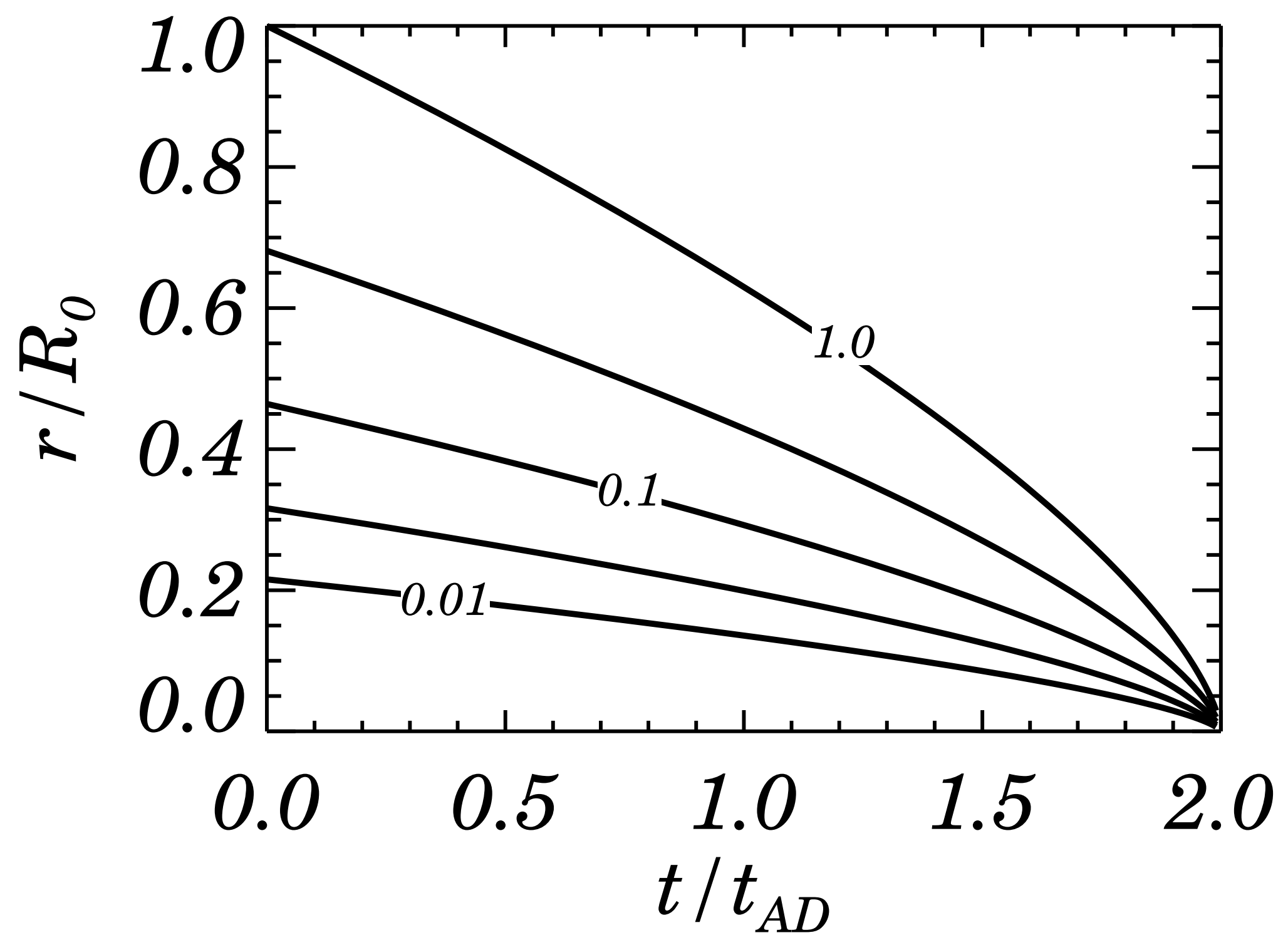

Figure 1 
(a)

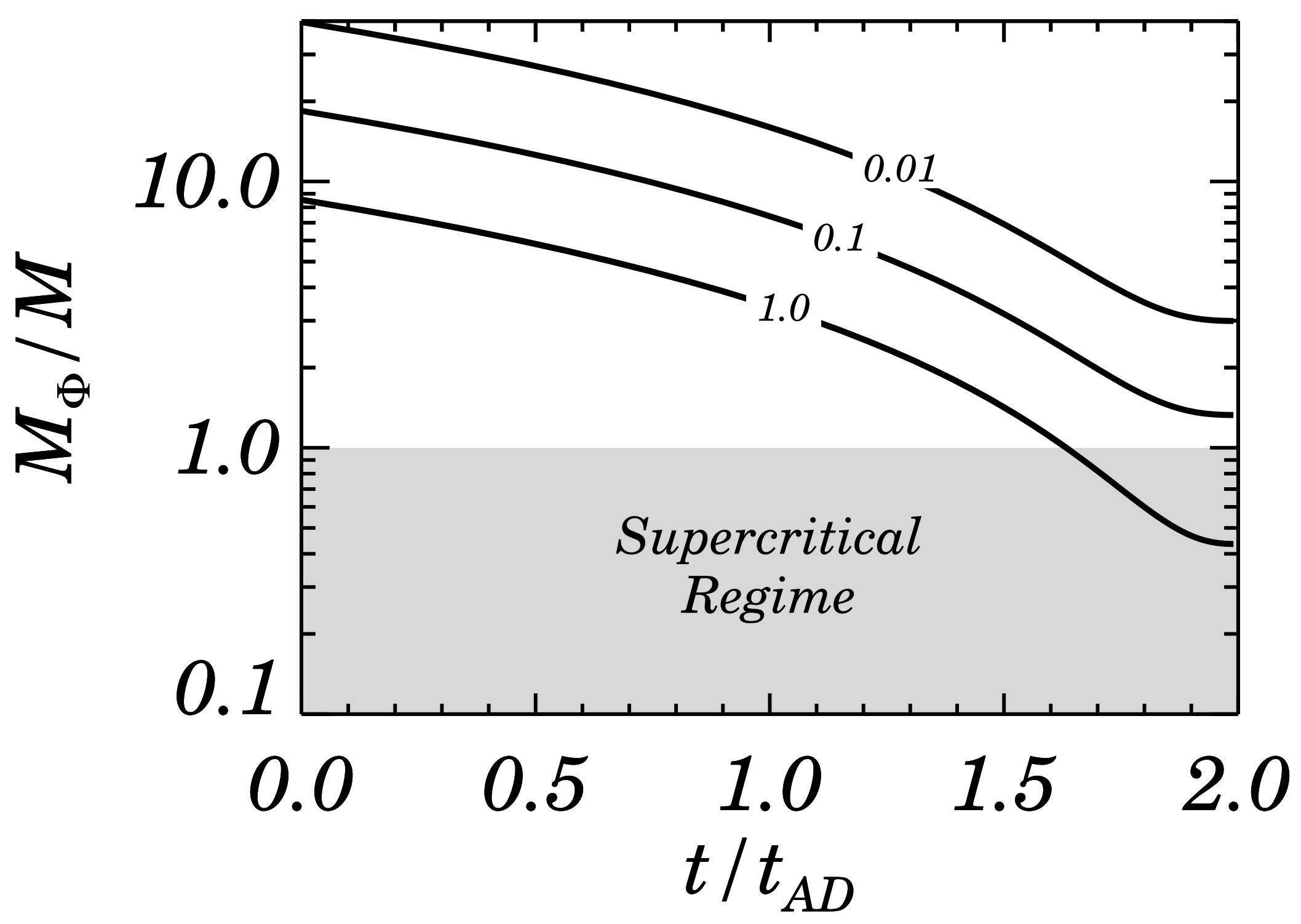

Figure 2a 
(b)

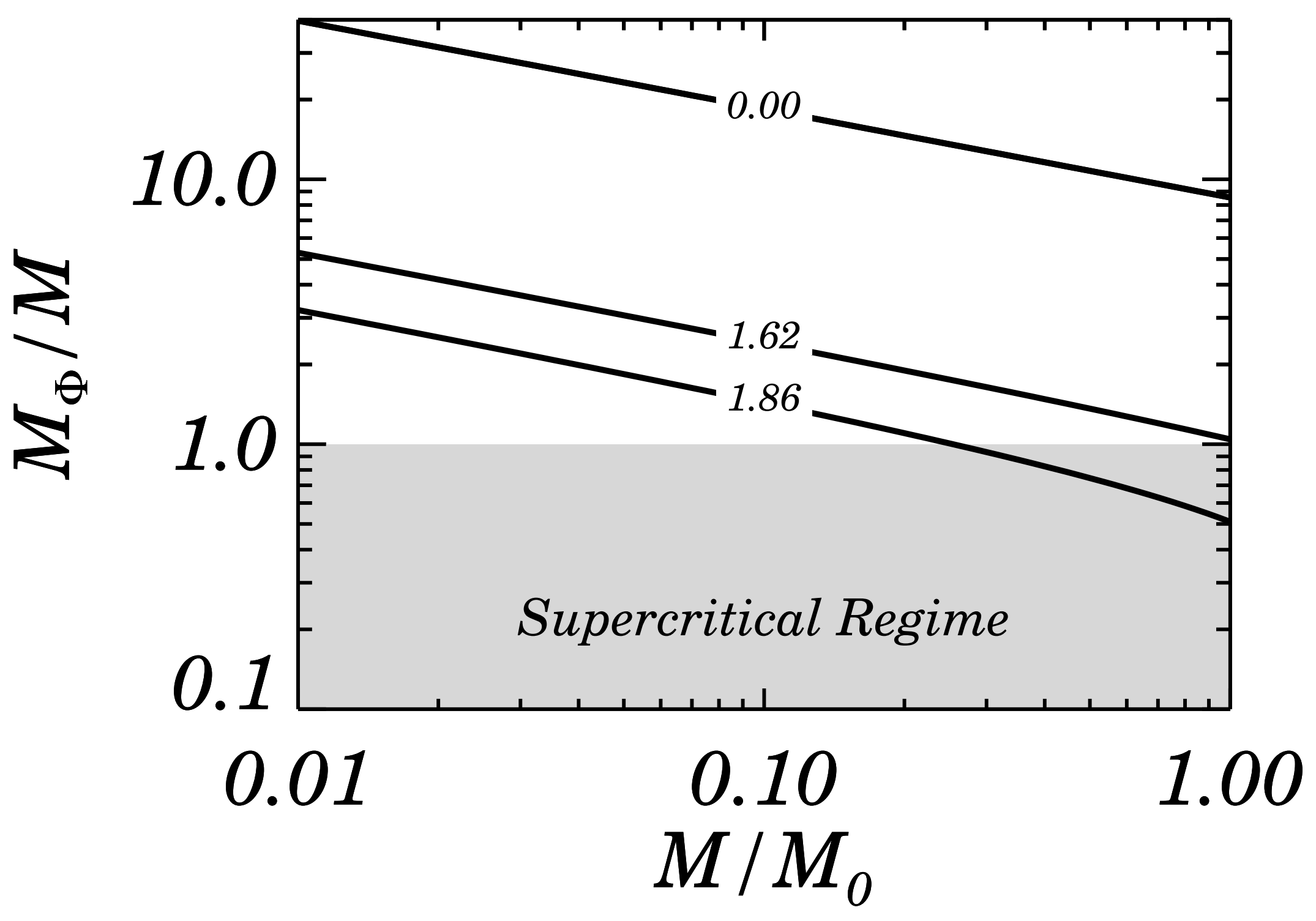

Figure 2b 


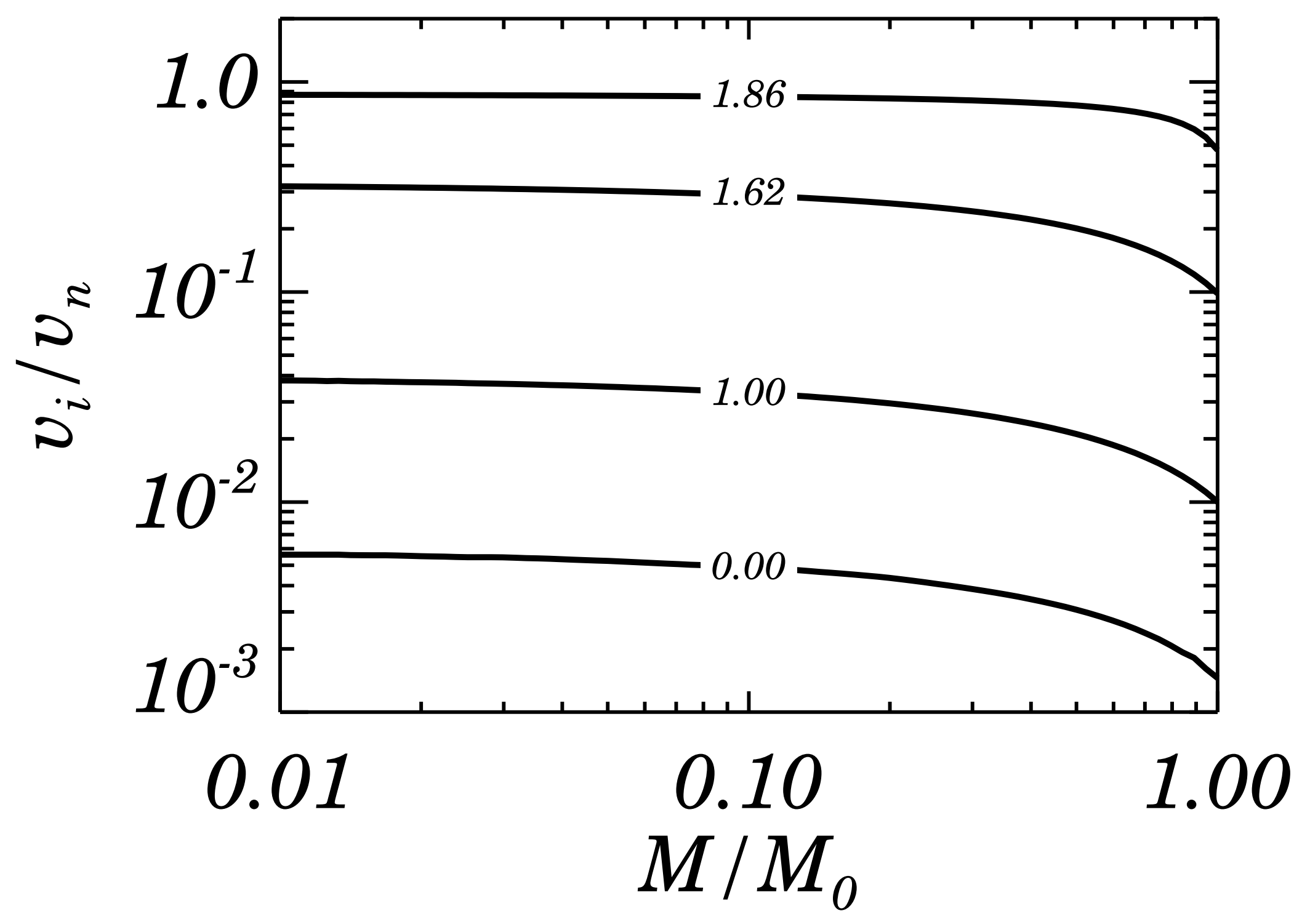

Figure 3 


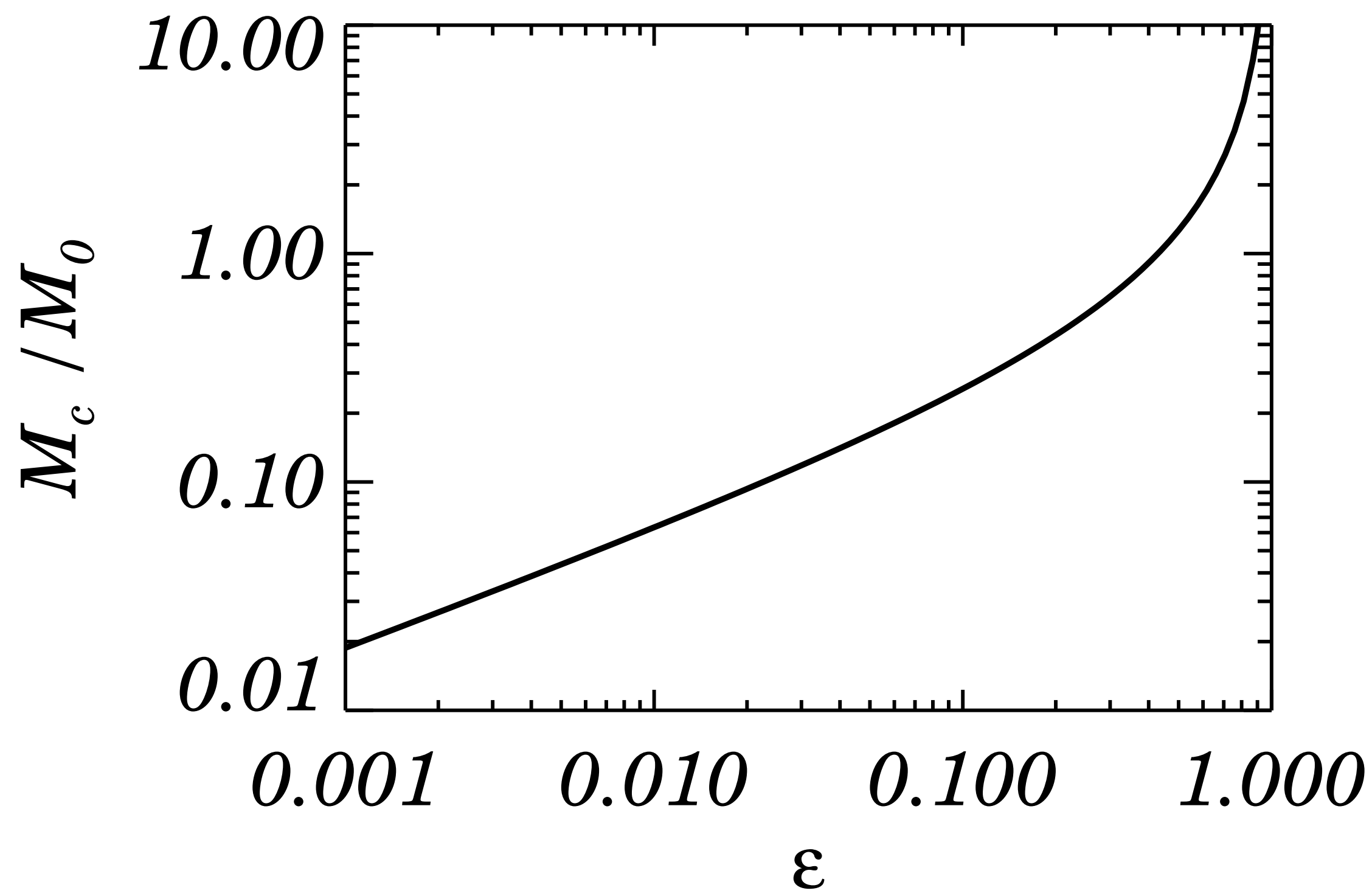

Figure 4 
(a)

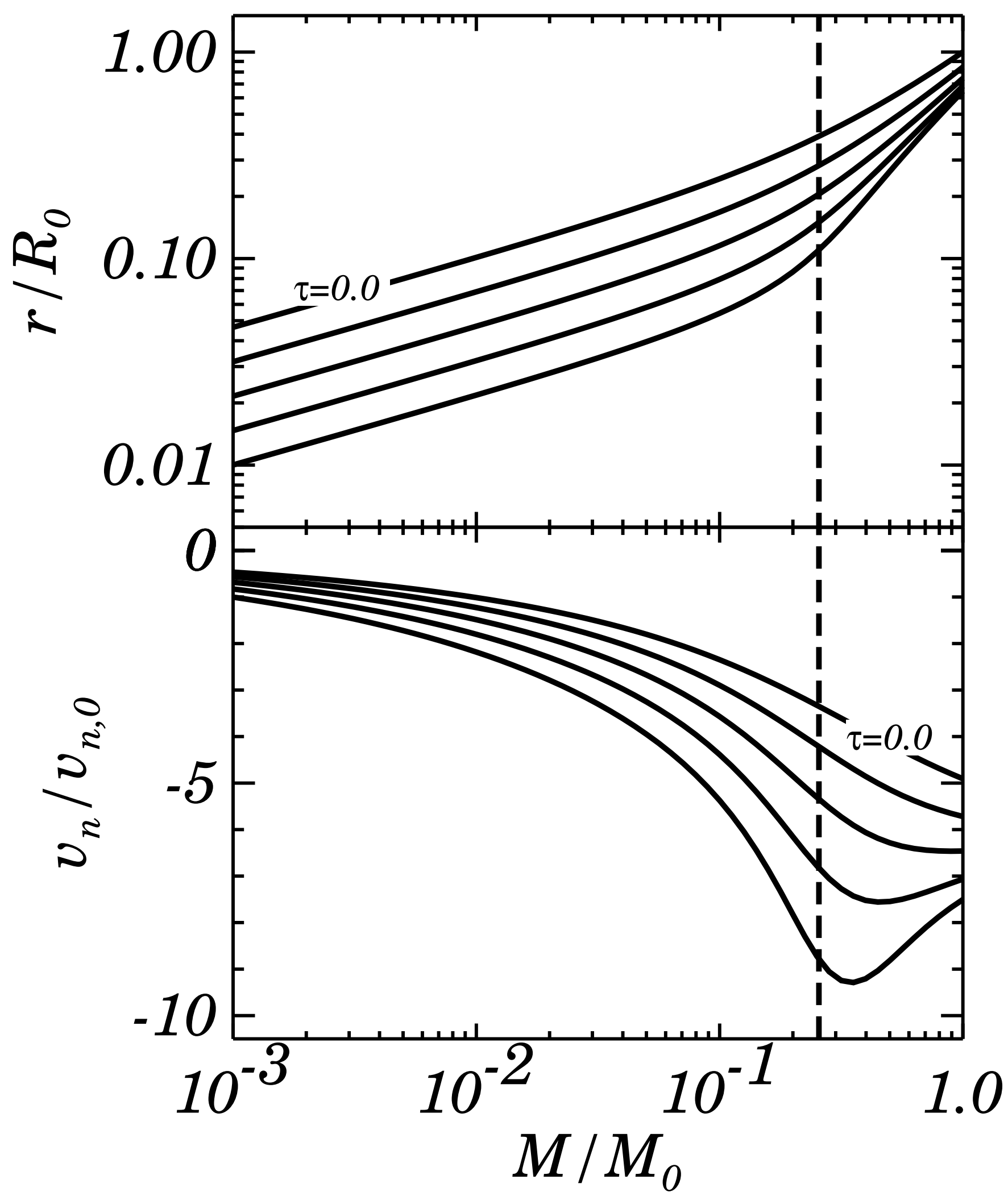

Figure 5a 
(b)

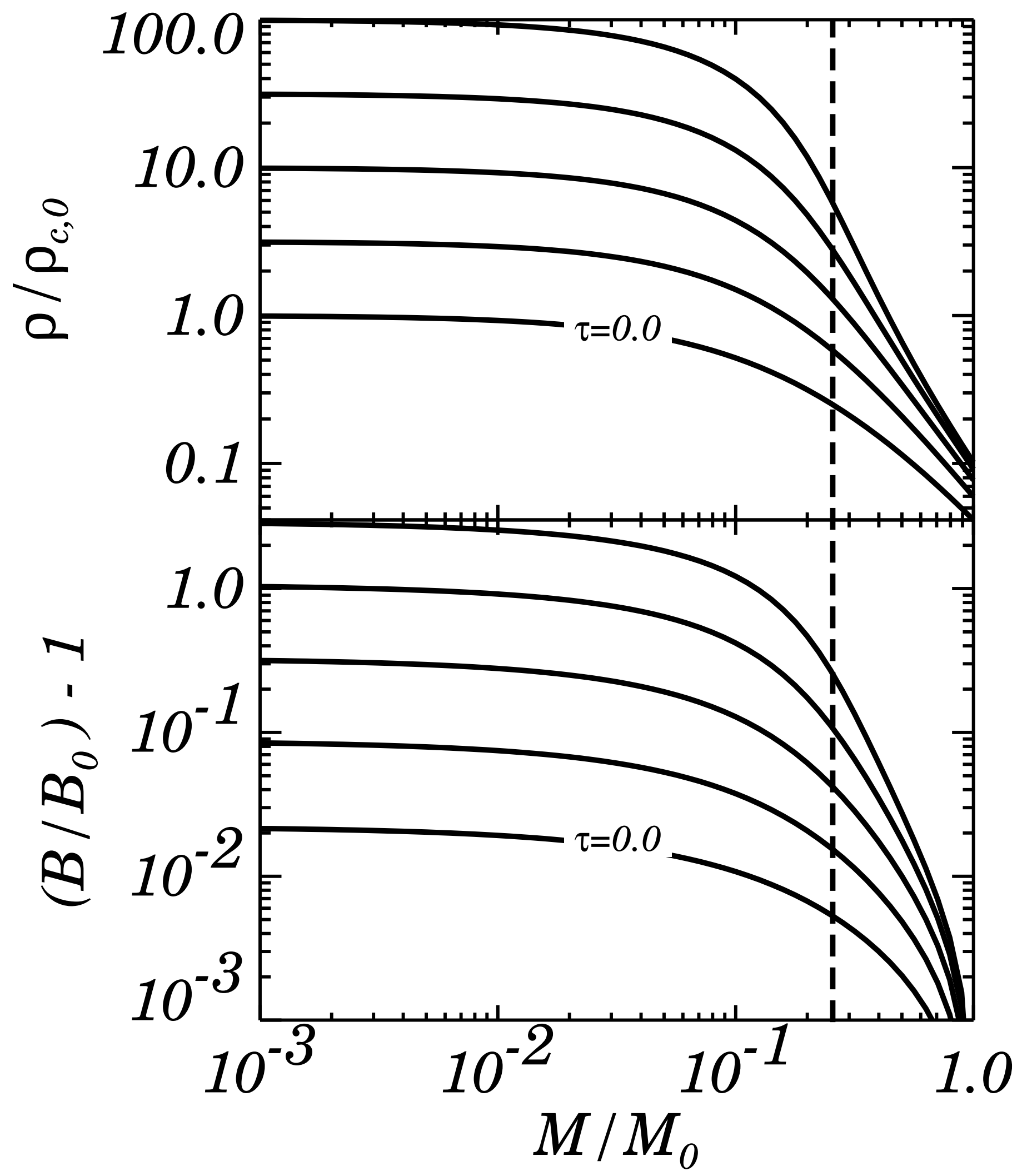

Figure 5b 


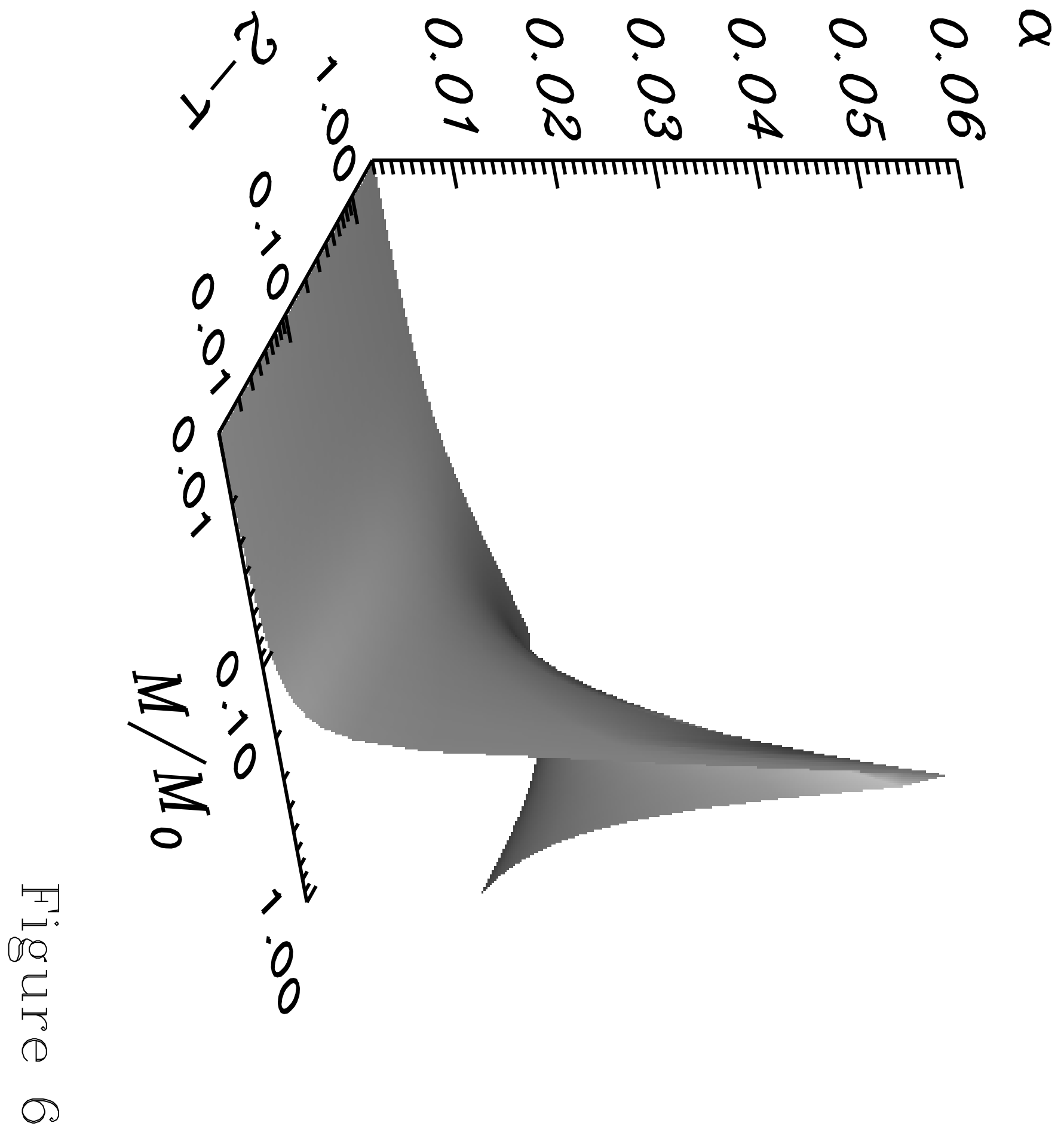




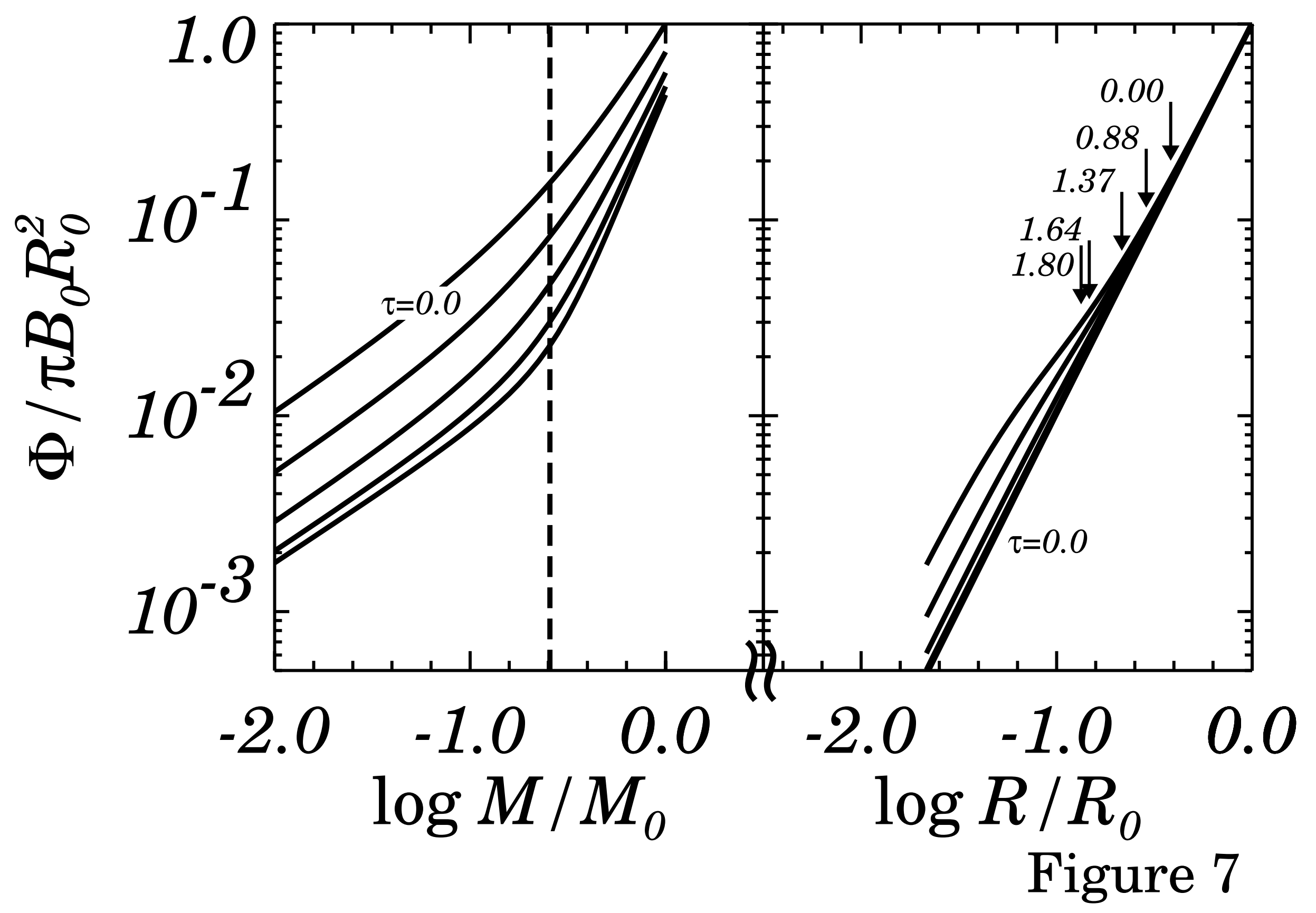




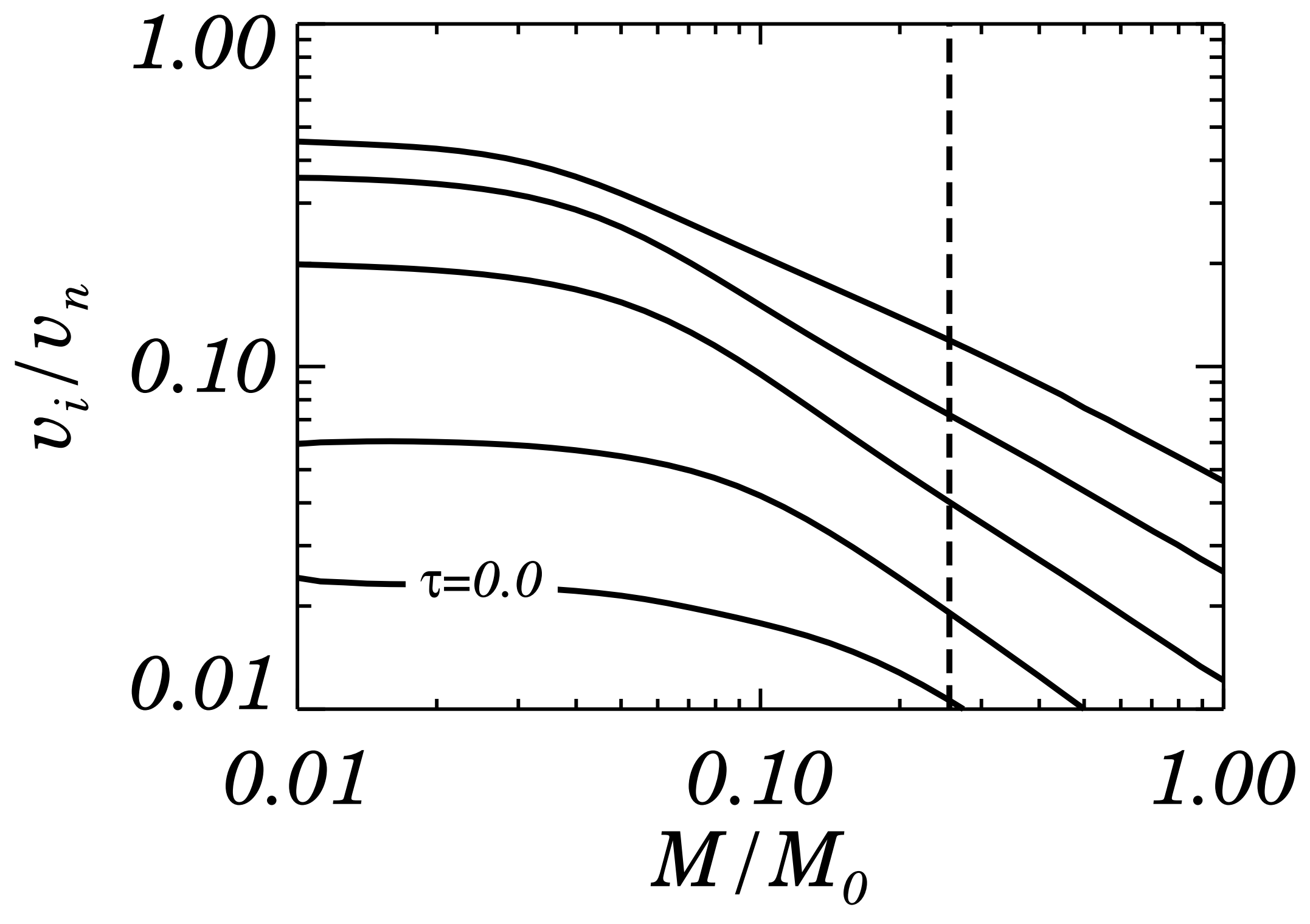

Figure 8 


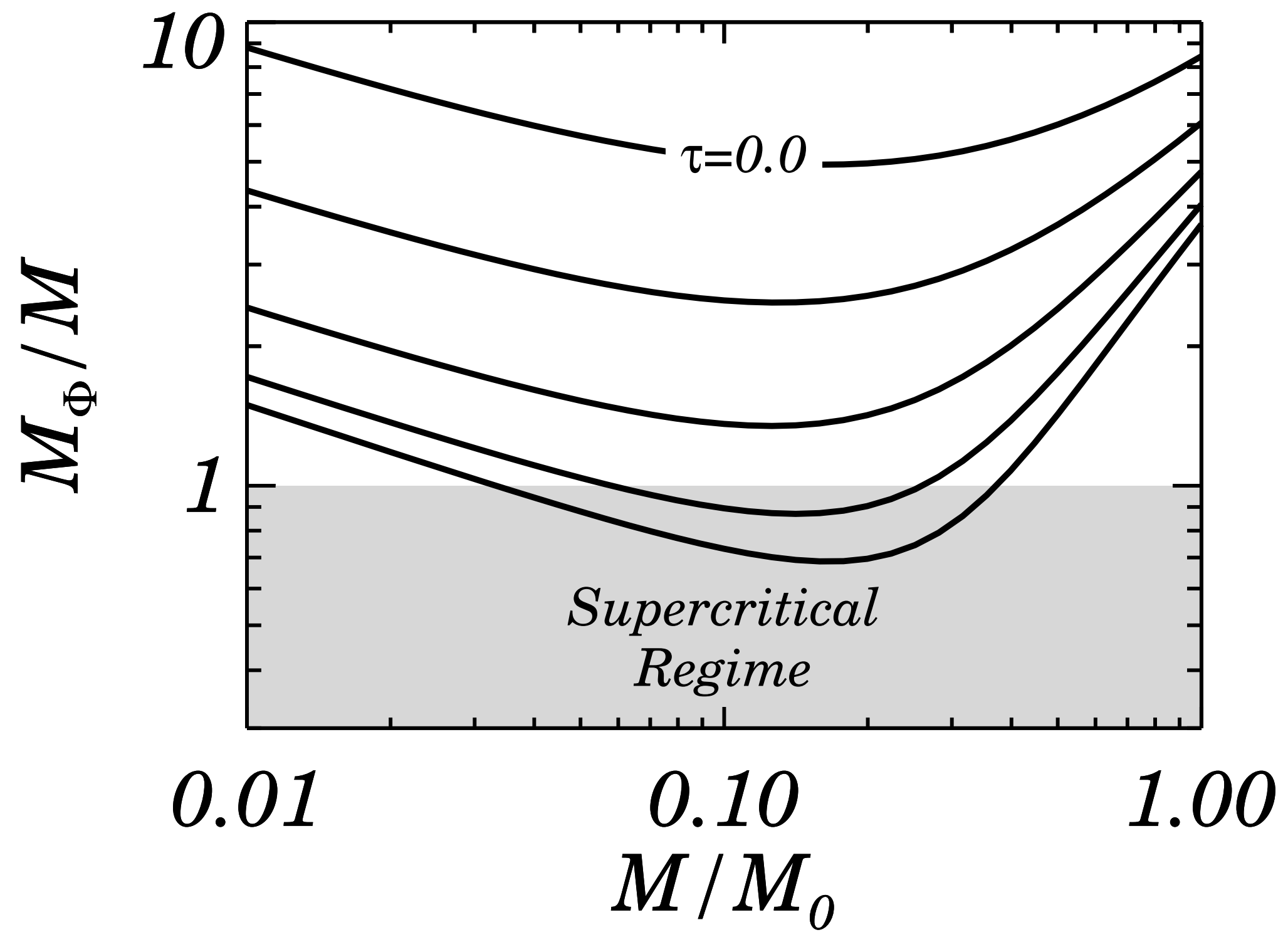

Figure 9 


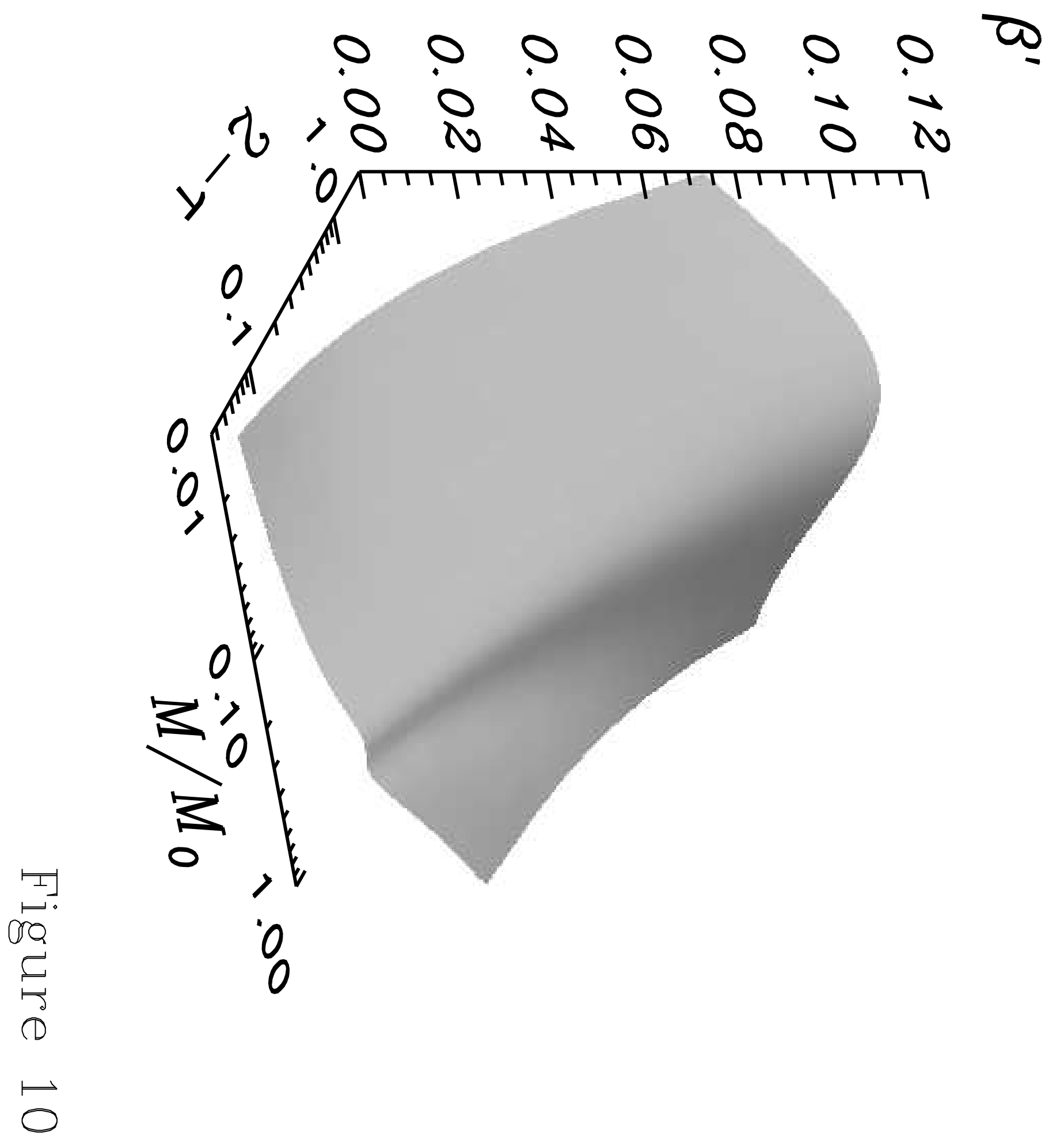




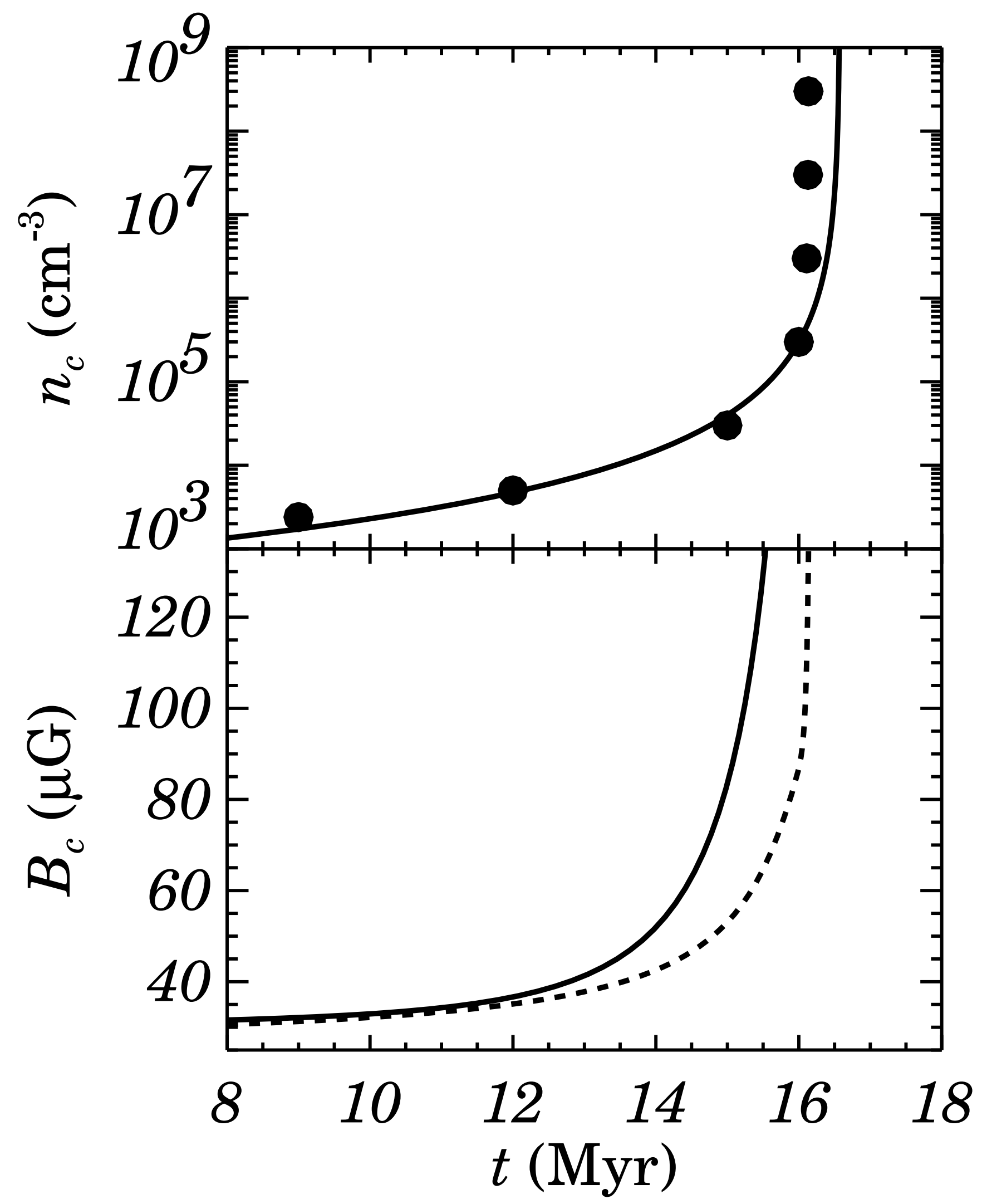

Figure 11 


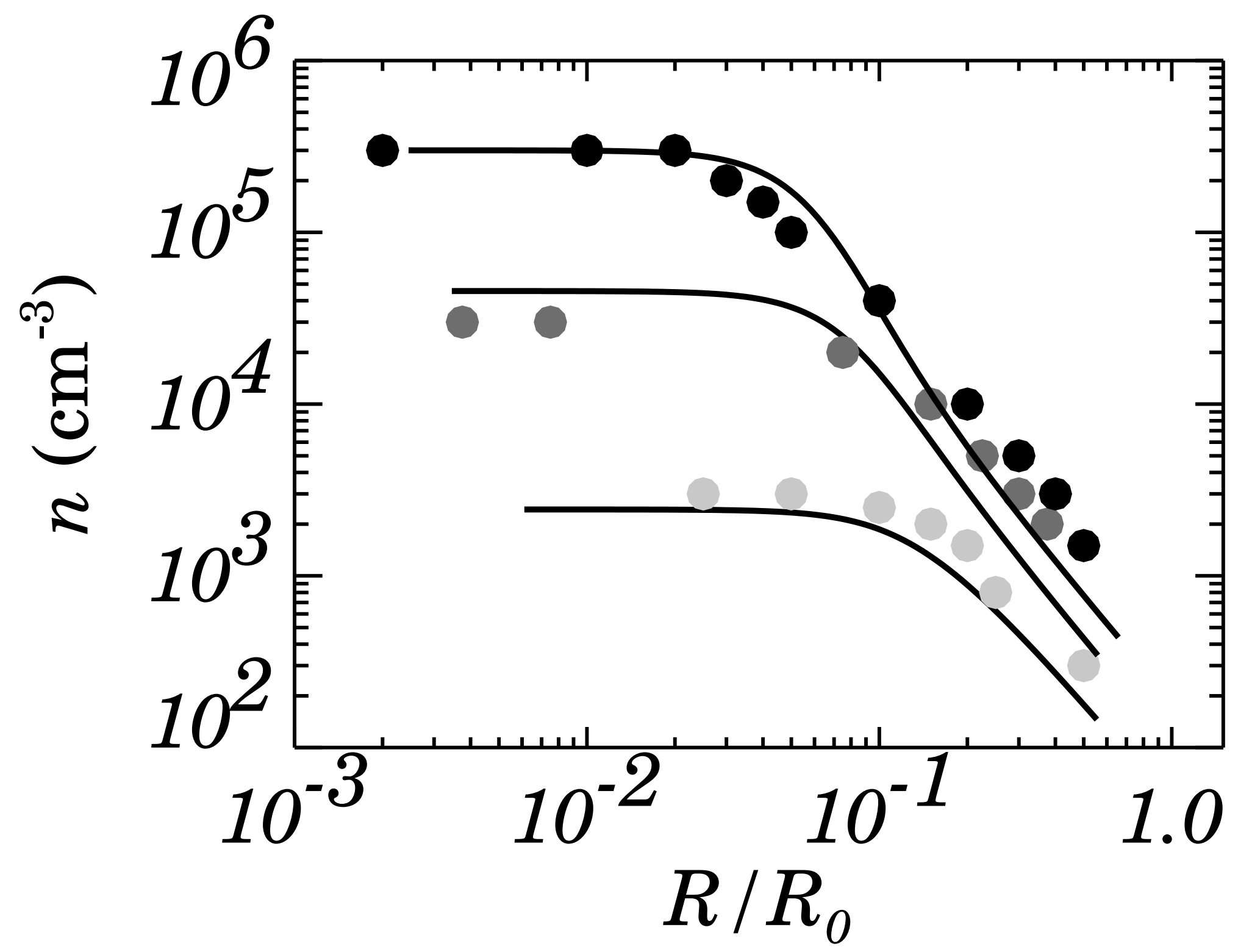

Figure 12 


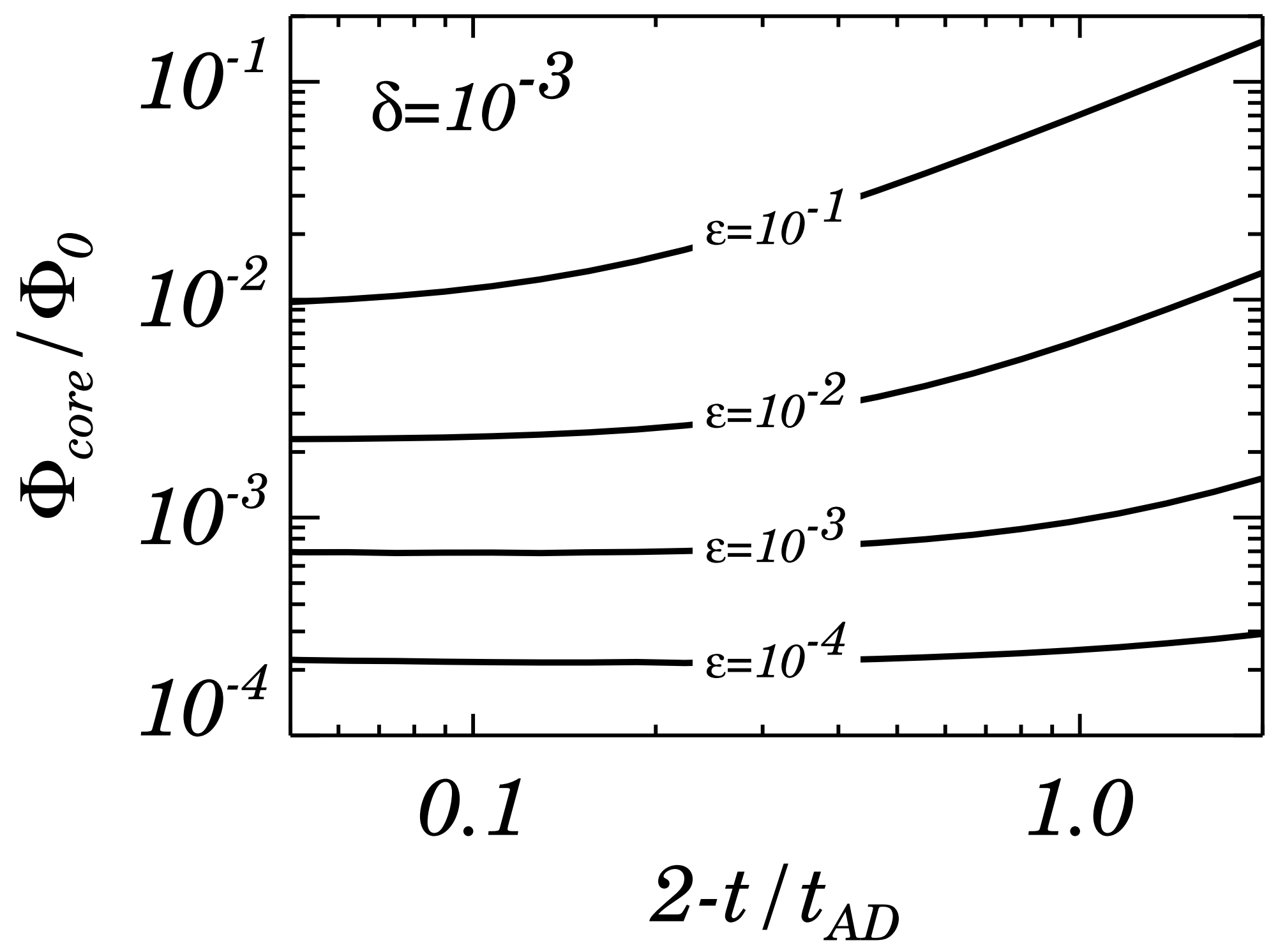

Figure 13 


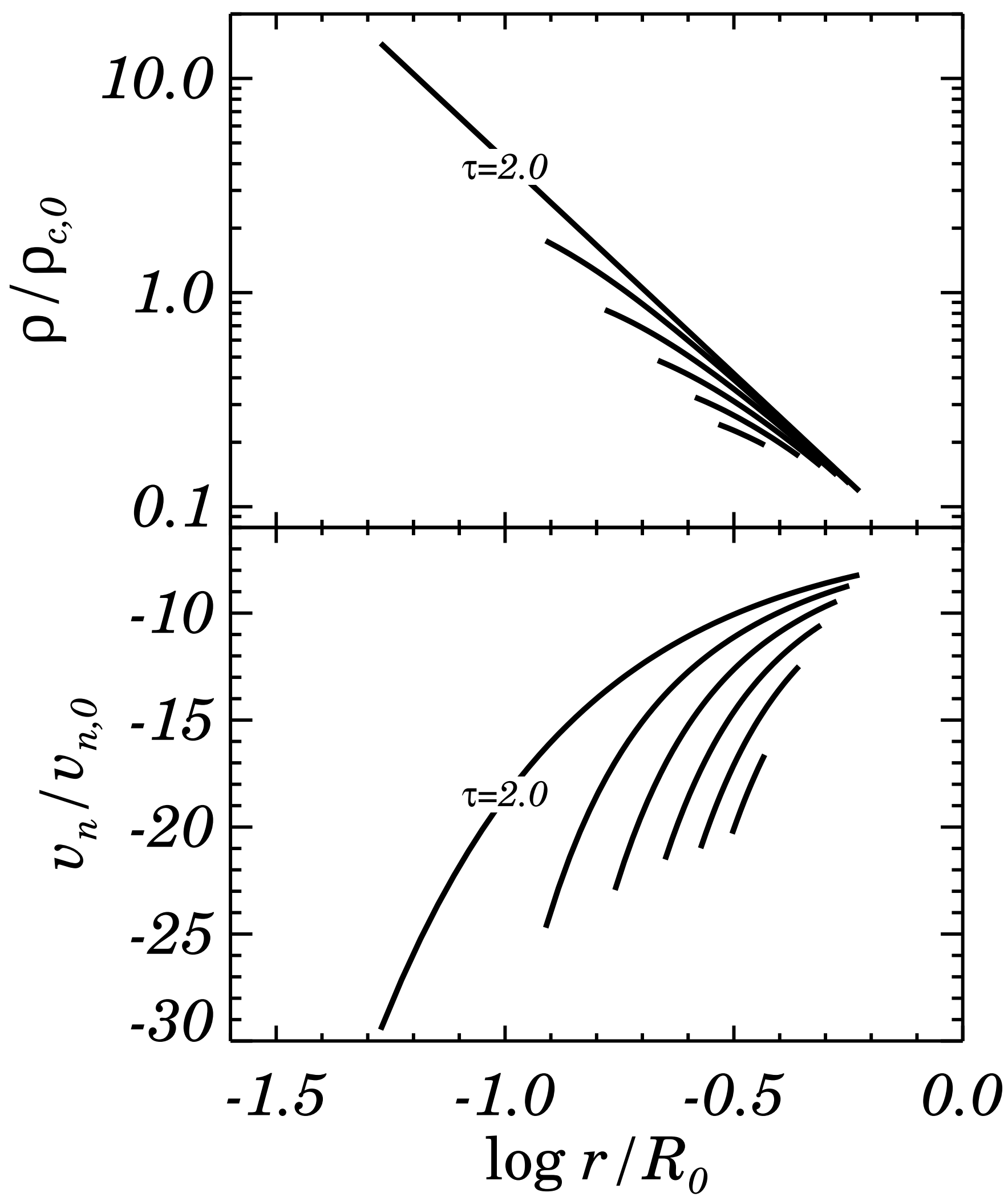

Figure 14 


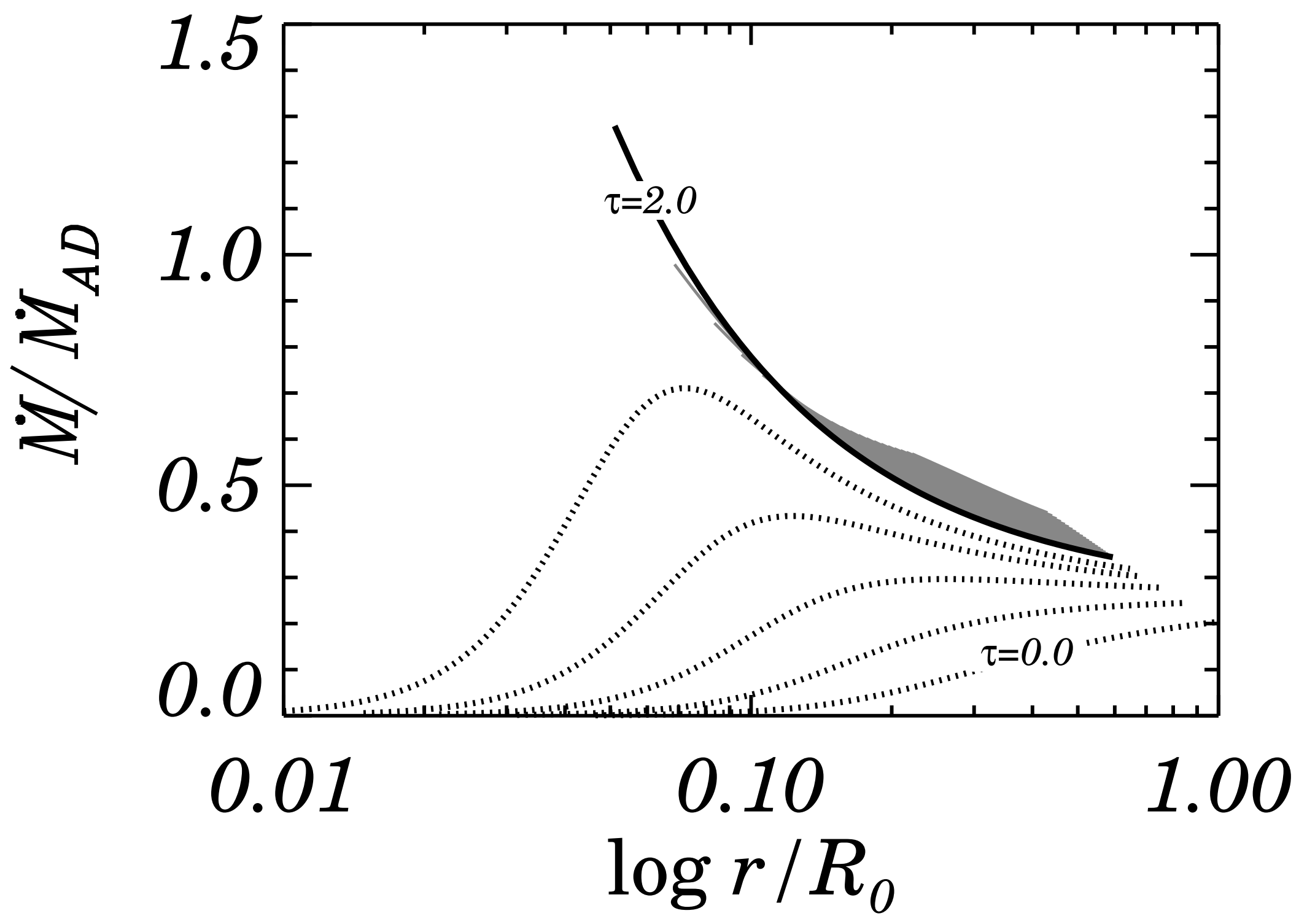

Figure 15 


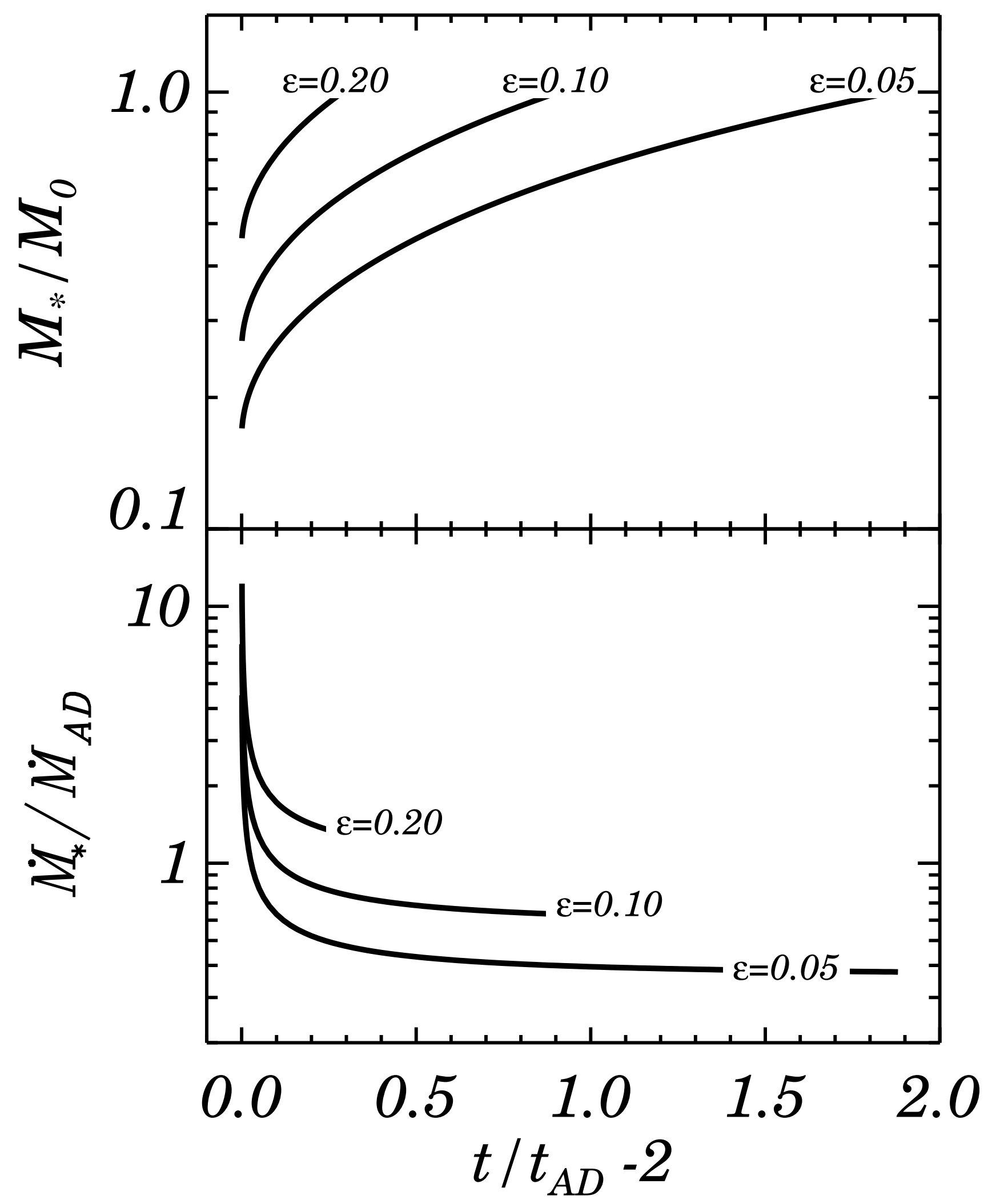

Figure 16 